\title{
Finite quantum field theory with rotatory quantization and gravitational regularization
}

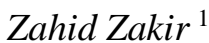

\begin{abstract}
At quantization of harmonic rotator, a rotational mode of planar oscillator, energy spectrum is linear on frequency and equidistant, but zero-point energy in ground state can not arise. This is in agreement with generalization of uncertainty relations to non-Hermitean canonical pairs. Quantization of waves at collective rotations of a chain of harmonic rotators allows one to model the fields with charge-conjugation and gauge symmetries. In quantum field theory (QFT) at quantization of rotational modes as harmonic rotators the observables of fields are normal ordered and zero-point energy and zero-point charge of vacuum do not arise. In this case frequencies of quanta are angular speeds of rotation of field vectors in real or field spaces and two signs of helicity correspond to a particle and an antiparticle. Photons with circular polarization and complex fields are examples of such fields, spin and isospins (charges) of particles can be related by their frequencies as angular momenta and helicities of the rotating field vectors. At rotational quantization of strings there are no zero-point energy of modes and here a conformal anomaly is absent, so spacetime dimensionality and gauge group are not fixed. In QFT the fields should be averaged in small cells of space and time, where distribution and evolution of fields are described classically, and field functions on borders of cells should be sewed. Then loop integrals are finite and the renormalized theories are invariant under reduction of the size of cells (a renormgroup with the cell regularization). The Planck scale cell is smallest because of freezing of proper times in a strong external gravitational field of the loop diagram with redshifting of frequencies up to zero. In the Standard Model and quantum gravity the loop contributions of fields, with exception of scalars, are small and the perturbation theory is convergent.
\end{abstract}

PACS: 03.65.Ge, 11.30.Er, 1130.Ly, 11.90. + t, 03.65.Ge, 03.70. + k, 11.10.Gh, 11.10.Hi, 11.15.Ha, 11.10.Ly, 12.20. - m, 11.25. - w, 12.10. - g,

Keywords: quantization, charge conjugation symmetry, harmonic rotator, quantum fields, vacuum energy, renormalization, regularization, strings, anomalies

\section{Content}

Introduction

1. Rotatory quantization of charge conjugation symmetric systems........................................4

1.1. Generalization of uncertainty relations and Poisson's brackets ................................. 4

1.2. Charge conjugation symmetric oscillators without zero-point energy..........................6

1.3. Negative norm problem for negative-frequency states and its solution ......................... 8

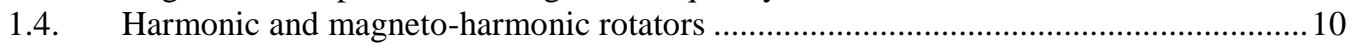

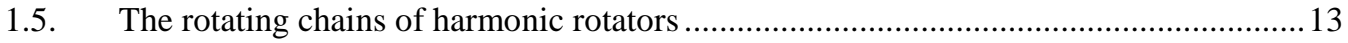

2. Rotatory quantization of C-symmetrical fields..........................................................16

2.1. Experimental bases for rotatory quantization ....................................................... 16

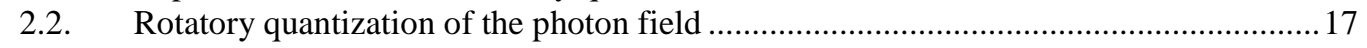

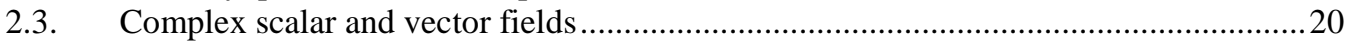

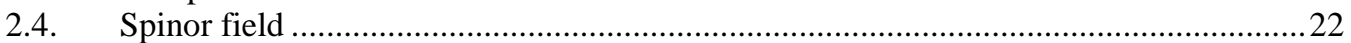

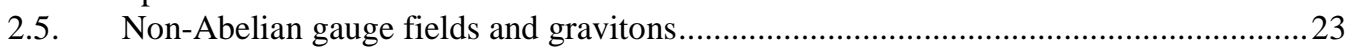

3. Quantum fields with natural regularizations ...........................................................................24

3.1. Temporary regularization of integrals on trajectories ...............................................24

${ }^{1}$ Centre for Theoretical Physics and Astrophysics, Tashkent, Uzbekistan, zahidzakir@theor-phys.org 
3.2. Field as continuous limit of a chain of harmonic rotators .........................................24

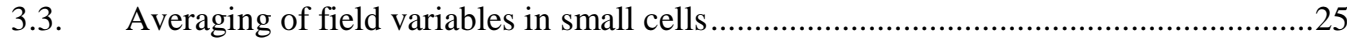

3.4. Natural regularizations of quantum fields and renormgroup ..................................25

3.5. Gravitational freezing of fluctuations at the Planck scale ..........................................27

3.6. Rotatory quantization and the Planck scale cutting of fields ......................................30

4. Particle physics: rotatory quantization and gravitational regularization .........................32

4.1. Finiteness and consistency of quantum electrodynamics ..........................................32

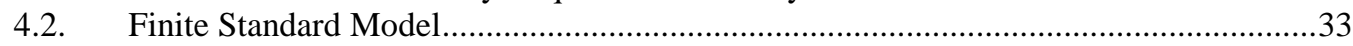

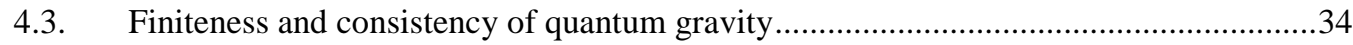

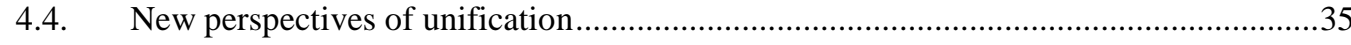

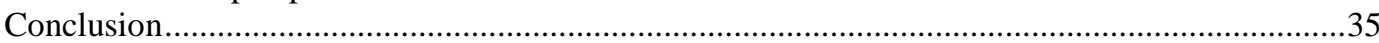

Appendix. Rotatory quantization of strings without anomalies ..................................................36

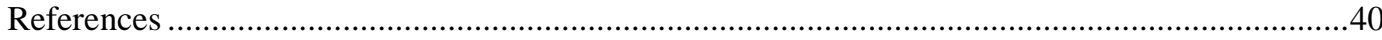

\section{Introduction}

In the standard formulation of quantum field theory (QFT) normal modes of relativistic fields and strings are quantized, firstly, by analogy with the linear harmonic oscillator having zero-point energy, and secondly, consequences of an external gravitational field of high energy quanta are ignored. All these led to divergences for zeropoint energy of vacuum and loop diagrams. When divergences were ruled out (cutted, «normally ordered», «renormalized»), the theory was became very successful, especially, in case of gauge fields [1-3]. Thus, the solution of these problems was shelved in hope for new hypotheses, but the latters created more problems, than solved [3].

In the papers [4-6] it was shown that QFT becomes consecutive and consistent without two stated above simplifications, while its formalism mostly conserves, which explains former success of the theory. Instead of restriction by vibrational modes of fields only, the theory has been constructed on the basis of their rotational modes. Thus, waves in the system appear as rotational waves, the modes of which are similar to the harmonic rotator - a rotational mode of the planar oscillator when a particle moves along circle. Spectrum of the harmonic rotator is equidistant and energy levels are linear on frequency.

Differences of rotational modes from vibrational ones lead to new physical properties of systems: a) frequency means the angular speed of rotation and states on rotation plane are two fold degenerated, b) angular momentum conserves and is quantized naturally, c) energy is proportional to angular momentum and, as the result, quantization of energy appears as a consequence of angular momentum quantization, d) there is no zeropoint energy from purely rotational modes.

In a chain of harmonic rotators collective rotations around a common axis lead at small perturbation to transverse waves which are quantized, and their quasiparticles and holes transfer the angular momentum. Thus, a symmetry group is not $O(2)$, as at an isolated rotator, but $S U(2)$, since the chain of rotators passes to itself not at rotation on $2 \pi$ of a single rotator, but at its rotation on $4 \pi$, when it is possible to untangle the chain at fixed external constraints. As a result, in the chain of rotators quanta in the spinor representation of rotation group can appear. Ladder operators in operators of observables are normally ordered, which expresses transition from vibrational to rotational modes.

Adequacy of treatment of fluctuations of fields as rotational ones is clearly shows the case of photons for which pure states are circularly polarized ones. Such photons are 
emitted by a rotating dipole when transverse vectors of the photon field are constant on module, rotate around the momentum direction, and the energy spectrum is equidistant. Thus, frequency is angular speed of rotation of field vectors, photon's spin is the angular momentum of this rotation, and the helicity is the conserving chiral charge. All these are exactly correspond to properties of quanta in the chains of harmonic rotators.

The relativistic fields, describing particles of the Standard Model (SM), are complex fields because of helicity states and existence of antiparticles, and their quanta in pure states are emitted by sources with transferring of angular momentum, spin or charge. Zero-point fluctuations of their vacuum was not revealed on experiments, and the effects ascribed them are caused in fact by real sources and exclude the zero-point energy of the vacuum. Therefore the theory of such fields should be constructed on the basis of harmonic rotator, where frequencies are angular speeds of a rotational mode of fields, and operators are always normally ordered.

Fields are represented usually as a continuous limit of a chain of oscillators, while in the developing approach the fields are considered as limits of chains of rotators. The fields also should be averaged in spatial cells $\Delta V$, small only with respect to a reached accuracy of measurements, and on boundaries of cells they should be continuously matched. Their time evolution is described classically in small time intervals $\Delta t$, smaller than a reached accuracy of time measurements. loop integrals then become finite, and the theories invariant under reduction of cell's size are renorm-invariant.

But for transition to very small size cells there is a fundamental physical restriction - the Planck scale $l_{p l}$ is a gravitational radius of quanta of Planck energy $\Lambda_{p l}$, where proper times freeze with respect to world time $t$ in which $S$-matrix is defined. Therefore the processes in cells smaller $l_{p l}$ do not contribute to transition amplitudes as requiring an infinite interval of $t$ and for finite world time simply can not occur.

Thus, the theory of relativistic fields should be constructed on the basis of rotatory quantization, not containing the zero-point energy, and reduction of cells, where field variables are averaged, is admissible only up to $l_{p l}$, so the theory does not contain ultraviolet divergences. loop contributions of gauge fields grow with energy logarithmically and at small bare charges are small up to $l_{p l}$, which makes the perturbation theory converging. Quantum gravity at gravitational regularization at $l_{p l}$ is finite and renormalizable.

In theories of strings and branes the zero-point energy of modes led to the anomaly and the central charge, and the requirement of lack of this anomaly fixed spacetime dimension and then restricted states and gauge symmetries. However, in [6] it was shown that at quantization of rotational modes of strings there is no zero-point energy, and thus the conformal anomaly does not arise and no restrictions on dimension of manifold. These results are presented in the Appendix.

In section 1 of the paper a generalization of uncertainty relations and the canonical formalism for complex variables are presented, a solution of the problem of negative norm of negative frequency states is described, and then the harmonic rotator and rotational waves in a chain of rotators are considered. In section 2 rotational quantization of charge conjugation symmetric fields is described, and in section 3 the cell regularization of QFT with lower limit at $l_{p l}$ is formulated. In section 4 applications of new treatment of QFT to SM and to quantum gravity are discussed. 


\section{Rotatory quantization of charge conjugation symmetric systems.}

\subsection{Generalization of uncertainty relations and Poisson's brackets}

The Heisenberg uncertainty relations are derived only for Hermitean operators for canonical variables. However, at quantization of charge conjugation symmetry systems we deal with non-Hermitean operators for canonical variables. In [4] it was described a generalization of these relations for non-Hermitean operators too.

Let mean values of canonical pairs $q, p$ and $q^{*}, p^{*}$ are zero. Then the following inequality takes place:

$$
\left\langle\left|\lambda q+\frac{i}{\hbar} p^{*}\right|^{2}\right\rangle=\left\langle\left(\lambda q^{*}-\frac{i}{\hbar} p\right)\left(\lambda q+\frac{i}{\hbar} p^{*}\right)\right\rangle \geq 0,
$$

where $\lambda$ is arbitrary real constant, and the inequality we can rewrite as:

$$
\left\langle q^{*} q\right\rangle \lambda^{2}+\frac{1}{\hbar}\left\langle i\left(q^{*} p^{*}-p q\right)\right\rangle \lambda+\frac{1}{\hbar^{2}}\left\langle p^{*} p\right\rangle \geq 0 .
$$

Inequality $a \lambda^{2}+b \lambda+c \geq 0$ at $a>0$ is satisfied for any $\lambda$ only at the condition $D=b^{2}-4 a c \leq 0$, which for the coefficients of Eq. (2) means an inequality:

$$
\left\langle q^{*} q\right\rangle\left\langle p^{*} p\right\rangle \geq \frac{1}{4}\left(i\left\langle q^{*} p^{*}-p q\right\rangle\right)^{2}=\frac{1}{4}\langle Q\rangle^{2} \text {. }
$$

In the right hand side there is a mean value of the charge operator $Q$ defined as:

$$
Q=i\left(q^{*} p^{*}-p q\right) \equiv[q, p]_{*} .
$$

If in the system with non-Hermitean operators the charge is quantized and its proper values are multiple to $\pm \hbar$, i.e. $\langle Q\rangle=\hbar n_{( \pm)}, n_{ \pm}=0, \pm 1, \pm 2, \ldots$, Eq. (3) turns to:

$$
\left\langle q^{*} q\right\rangle\left\langle p^{*} p\right\rangle \geq \frac{\hbar^{2}}{4} n_{ \pm}^{2} \text {. }
$$

The relations (3)-(5) are generalizations of uncertainty relations on the systems with non-Hermitean operators of canonical pairs. Expressions for nonzero mean values can be obtained by shifts $q^{\prime}=q-\langle q\rangle, \quad p^{\prime}=p-\langle p\rangle$.

Right hand side of Eq. (3) can be presented also through proper values of the angular momentum operator $M$, using its relation by $Q$ :

$$
M=q_{1} p_{2}-q_{2} p_{1}=i\left(q p-q^{*} p^{*}\right)=-i\left(q^{*} p^{*}-p q\right)-i(p q-q p)=-(Q+1) .
$$

Thus, the inequality (3) takes the form:

$$
\left\langle q^{*} q\right\rangle\left\langle p^{*} p\right\rangle \geq \frac{1}{4}(\hbar+\langle M\rangle)^{2} .
$$

For the real canonical variables we have $\langle M\rangle=0$ and the expression $q^{*} p^{*}-p q$ turns to the ordinary commutator $(q p-p q)=i \hbar$. It then leads to the standard Heisenberg uncertainty relations. If at charge conjugation symmetry for the ground state $\langle Q\rangle_{0}=0$, in accordance with (5) we obtain:

$$
\left\langle q^{*} q\right\rangle_{0}\left\langle p^{*} p\right\rangle_{0} \geq 0
$$

It takes place at $\langle M\rangle=-\hbar$, and also for relativistic fields with charge conjugation symmetry and in their ground state there is no "zero-point energy". 
Notice that new relations impose stronger restrictions on the fluctuations of canonical variables - a minimum lies as higher as larger the charge or angular momentum. In the nonrelativistic theory it is caused by the fact that for the energy of rotations, associated with the angular momentum, the minimum of system's effective potential lies as higher as larger the centrifugal energy. The situation is similar for the charge conjugation symmetric systems.

At usual definition of the Poisson brackets, as a rule, for two pairs of canonical variables only two brackets:

$$
\{A, B\}_{P B} \equiv \frac{\partial A}{\partial p} \frac{\partial B}{\partial q}-\frac{\partial A}{\partial q} \frac{\partial B}{\partial p}, \quad\{A, B\}_{P B^{*}}=\frac{\partial A}{\partial p^{*}} \frac{\partial B}{\partial q^{*}}-\frac{\partial A}{\partial q^{*}} \frac{\partial B}{\partial p^{*}}
$$

have been considered only. Each of them is invariant under one of two groups of canonical transformations: $(p, q) \rightarrow(P, Q),\left(p^{*}, q^{*}\right) \rightarrow\left(P^{*}, Q^{*}\right)$. In fact in such complex variable systems it is possible to consider the mixed brackets too:

$$
\begin{aligned}
& \{A, B\}_{*} \equiv \frac{\partial A}{\partial p^{*}} \frac{\partial B}{\partial q^{*}}-\frac{\partial A}{\partial q} \frac{\partial B}{\partial p}, \quad\{A, B\}_{m 1} \equiv \frac{\partial A}{\partial p} \frac{\partial B}{\partial q}-\frac{\partial A}{\partial q^{*}} \frac{\partial B}{\partial p^{*}}, \\
& \{A, B\}_{m 2} \equiv \frac{\partial A}{\partial p} \frac{\partial B}{\partial q}-\frac{\partial A}{\partial p^{*}} \frac{\partial B}{\partial q^{*}}, \quad\{A, B\}_{m 3} \equiv \frac{\partial A}{\partial q} \frac{\partial B}{\partial p}-\frac{\partial A}{\partial q^{*}} \frac{\partial B}{\partial p^{*}} .
\end{aligned}
$$

In case of real generalized coordinates the first two mixed brackets pass into ordinary Poisson brackets and the last two, changing a sign at replacements $q \rightarrow q^{*}, p \rightarrow p^{*}$, disappear. Six types of brackets (9)-(10) are related by three identities:

$$
\begin{aligned}
& \{A, B\}_{*}+\{A, B\}_{m 1}=\{A, B\}_{P B}+\{A, B\}_{P B^{*}}, \\
& \{A, B\}_{m 2}=\{A, B\}_{*}-\{A, B\}_{P B^{*}}, \quad\{A, B\}_{m 3}=\{A, B\}_{P B^{*}}-\{A, B\}_{m 1} .
\end{aligned}
$$

Therefore, only three of them are independent and as such we can take two standard ones (9) and one of the mixed brackets (10), for example $\{A, B\}_{*}$.

The physical sense of the third independent bracket is related with the canonical invariant of rotational symmetry on the complex plane of $q$. This invariant is the charge $Q$ at rotations in space of complex variables and angular momentum $M$ at rotations in real space.

For the generalized coordinates and momenta we have:

$$
\begin{gathered}
\left\{p_{i}, q_{j}\right\}_{P B}=\left\{p_{i}^{*}, q_{j}^{*}\right\}_{P B^{*}}=\delta_{i j},\left\{p_{i}, q_{j}\right\}_{*}=\left\{p_{i}, q_{j}\right\}_{m 1}=-\left\{p_{i}^{*}, q_{j}^{*}\right\}_{m 1}=\delta_{i j} \\
\left\{p_{i}, q_{j}\right\}_{*}=\left\{p_{i}, q_{j}\right\}_{m 1}=-\left\{p_{i}^{*}, q_{j}^{*}\right\}_{m 1}=\delta_{i j} .
\end{gathered}
$$

Then, in addition to ordinary relations:

$$
\{A, q\}_{P B}=\frac{\partial A}{\partial p}, \quad\{p, B\}_{P B}=\frac{\partial B}{\partial q},\left\{A, q^{*}\right\}_{P B^{*}}=\frac{\partial A}{\partial p^{*}}, \quad\left\{p^{*}, B\right\}_{P B^{*}}=\frac{\partial B}{\partial q^{*}},
$$

following new ones should be added:

$$
\begin{gathered}
\{A, q\}_{*}=\frac{\partial A}{\partial p}, \quad\{p, B\}_{*}=\frac{\partial B}{\partial q},\left\{q^{*}, B\right\}_{*}=-\frac{\partial B}{\partial p^{*}},\left\{A, p^{*}\right\}_{*}=-\frac{\partial A}{\partial q^{*}} \\
\{q, B\}_{m 1}=-\frac{\partial B}{\partial p},\{A, p\}_{m 1}=-\frac{\partial B}{\partial q},\left\{A, q^{*}\right\}_{m 1}=\frac{\partial A}{\partial p^{*}},\left\{p^{*}, B\right\}_{m 1}=\frac{\partial B}{\partial q^{*}} .
\end{gathered}
$$

These relations can be used at integration of the equations of motion. 
If the standard commutators $[A, B]$ are quantum analogs of the standard Poisson brackets $\{A, B\}_{P B}$, here the generalized angular momentum (charge) $Q$ is a quantum analog of the mixed bracket $\{A, B\}_{*}$. Entering the mixed commutator corresponding to this bracket $[A, B]_{*}$ :

$$
[A, B]_{*}=i\left(A^{*} B^{*}-B A\right),
$$

the charge operator can be expressed through it as:

$$
Q=[q, p]_{*} \text {. }
$$

For the real generalized coordinates $Q$ passes into the ordinary commutator:

$$
Q \rightarrow i(q p-p q)=[q, p] .
$$

\subsection{Charge conjugation symmetric oscillators without zero-point energy}

Relativistic fields, except neutral ones, were represented as sets of harmonic oscillators with the complex generalized coordinates $q, q^{*}$ and momenta $p, p^{*}$. The symmetry under rotations in the complex plane led to the conserving charge $Q$. In [4] it was shown that if states with different charges are related by the charge-conjugation symmetry with the charge-conjugation operator $C$, there appear new restrictions.

Frequency decomposition of canonical variables has the form:

$$
\begin{aligned}
& q=\frac{1}{\sqrt{2 m \omega}}\left(a e^{-i \omega t}+\beta^{*} e^{i \omega t}\right), \quad q^{*}=\frac{1}{\sqrt{2 m \omega}}\left(a^{*} e^{i \omega t}+\beta e^{-i \omega t}\right), \\
& p=\frac{i m \omega}{\sqrt{2 m \omega}}\left(a^{*} e^{i \omega t}-\beta e^{-i \omega t}\right), \quad p^{*}=\frac{-i m \omega}{\sqrt{2 m \omega}}\left(a e^{-i \omega t}-\beta^{*} e^{i \omega t}\right) .
\end{aligned}
$$

Hamiltonian $H$ and the charge $Q$ of the system usually are taken in the form:

$$
\begin{gathered}
H=\frac{1}{m} p p^{*}+m \omega^{2} q^{*} q=\omega\left(a^{*} a+\beta \beta^{*}\right) . \\
Q=i\left(q^{*} p^{*}-p q\right)=a^{*} a-\beta \beta^{*},
\end{gathered}
$$

The commutators $i[p, q]=i\left[p^{*}, q^{*}\right]=1$ lead to:

$$
\left[a, a^{*}\right]=\left[\beta, \beta^{*}\right]=1 \text {. }
$$

In the Hamiltonian (21) and the charge operator (22) it has been accepted to rearrange the second pair of operators in a normal ordered form $\beta \beta^{*}=\beta^{*} \beta+1$ and then there appeared the zero-point energy and the zero-point charge of two types of oscillators. Thus the $C$-symmetry, which was exact before quantization, became broken by a quantum correction - the ground state zero-point charge. This situation has some features of a quantum anomaly (symmetry is broken by the quantum correction), and spontaneous symmetry breaking (the Hamiltonian is symmetrical, while the ground state does not).

However, operators $\beta, \beta^{*}$ are not «charge-conjugate» to the operators $a, a^{*}$ of «main» quanta [4]. If in the system $C$-symmetry is exact, $\beta, \beta^{*}$ it is necessary to express through operators $b, b^{*}$, entered as «charge-conjugate» to $a, a^{*}$.

At lack of an external field there is degeneration of energy levels under two values of the charge, so at $C$ - conjugation $H$ does not change, while the charge changes a sign:

$$
H^{c} \equiv C H C^{-1}=H, \quad Q^{c} \equiv C Q C^{-1}=-Q .
$$

The ladder operators, charge-conjugate to former ones, are defined as: 


$$
\begin{gathered}
b=C a C^{-1}, \quad b^{*}=C a^{*} C^{-1}, \quad \alpha=C \beta C^{-1}, \quad \alpha^{*}=C \beta^{*} C^{-1} . \\
{\left[b, b^{*}\right]=\left[\alpha, \alpha^{*}\right]=1 .}
\end{gathered}
$$

Then for the charge-conjugate states the Hamiltonian and the charge take the form:

$$
H^{c}=\omega\left(b^{*} b+\alpha \alpha^{*}\right), \quad Q^{c}=b^{*} b-\alpha \alpha^{*} .
$$

The $C$-symmetry requirements (24) in a detailed form represent the relations:

$$
\begin{aligned}
& H=\omega\left(a^{*} a+\beta \beta^{*}\right)=\omega\left(b^{*} b+\alpha \alpha^{*}\right)=H^{c}, \\
& Q=a^{*} a-\beta \beta^{*}=-\left(b^{*} b-\alpha \alpha^{*}\right)=-Q^{c}
\end{aligned}
$$

which mean two equations for four bilinear operator products:

$$
\begin{aligned}
& a^{*} a+\beta \beta^{*}=b^{*} b+\alpha \alpha^{*}, \\
& a^{*} a-\beta \beta^{*}=-b^{*} b+\alpha \alpha^{*} .
\end{aligned}
$$

By adding and subtracting these two relations, we obtain two operator identities:

$$
\alpha \alpha^{*}=a^{*} a, \beta \beta^{*}=b^{*} b,
$$

where the second identity is the charge-conjugate form of the first one. These identities allow one to exclude from the Hamiltonian and the charge the auxiliary operators $\beta, \beta^{*}$ and $\alpha, \alpha^{*}$, by expressing they through the operators $a, a^{*}$ of the basic quanta and chargeconjugate to them quanta $b, b^{*}$ :

$$
H=H^{c}=\omega\left(a^{*} a+b^{*} b\right), \quad Q=-Q^{c}=a^{*} a-b^{*} b .
$$

As we see, operators the observables are normally ordered and in the systems with exact $C$-symmetry there is no zero-point energy and zero-point charge.

The charge $Q$ is an analog of angular momentum $M$, but for rotations in the complex plane $q$. For ordinary rotations the energy is expressed through angular momentum as $H=M \omega$, where $\omega$ is angular speed. By analogy we will present $Q$ as the sum of charge operators for two types of oscillators:

$$
Q=-Q^{c}=Q_{a}+Q_{b}, \quad Q_{a}=a^{*} a, \quad Q_{b}=b^{*} b .
$$

Then the Hamiltonian (28) can be written through these charge operators in the form:

$$
H=H^{c}=Q_{a} \omega-Q_{b} \omega .
$$

These relations, testify that physical interpretation of disappearance of zero-point energy for oscillators with $C$-symmetry can be related by some rotations, let in a «charge space».

Lack of the zero-point energy for oscillators with $C$-symmetry unusual for the nonrelativistic systems. But generalization of the nonrelativistic harmonic oscillator model on relativistic fields is in fact purely formal and therefore such consequence of the formalism, as lack of the zero-point energy for relativistic systems, should be considered as a new prospect for investigations.

At quantization of the linear oscillator the zero-point energy appears also from the uncertainty relations. Generalization of these relations on the systems with non-Hermitean canonical pairs (3) shows that if the ground state is $C$-symmetric and $\langle Q\rangle=0$, there is no zero-point energy too.

At considering of interactions of $C$-symmetric systems of oscillators we take the $C$-symmetric interaction potentials only. At the complex generalized coordinate $q$ and potential $V\left(q^{*} q\right)$ there is $U(1)$ symmetry with the operator $U=\exp (i Q \alpha), \alpha=$ const. and the charge is conserved: $\partial_{t} Q=0$. At rotational symmetry $U(t)=\exp [i Q \alpha(t)]$ with 
a time-dependent parameter $\alpha(t)$ the presence of a gauge field is necessary $A_{\mu}=(\phi, \mathbf{A})$, transforming as $A_{\mu}{ }^{\prime}=A_{\mu}+\partial_{\mu} \alpha$, and there appears a covariant time derivative $D_{t} \equiv \partial_{t}-i g \phi$, where $g$ is an interacting constant.

The minimum form of a gauge-invariant Lagrangian has the form:

$$
L=m\left(D_{t} q\right)^{*}\left(D_{t} q\right)-V=m\left(\partial_{t} q\right)^{*}\left(\partial_{t} q\right)+g Q \phi+m g^{2} \phi^{2} q^{*} q-V,
$$

At quantization the generalized momenta $\pi^{*}(t)=m\left(D_{t} q\right)$ and $\pi(t)=m\left(D_{t} q\right)^{*}$ should mutually commutate, but then speeds $\partial_{t} q$ and $\left(\partial_{t} q\right)^{*}$ do not commutate:

$$
\left[\pi(t), \pi^{*}(t)\right]=0, \quad\left[\partial_{t} q(t), \partial_{t} q^{*}(t)\right]=\frac{2}{m} g \phi M,
$$

where the effective «angular momentum» $M$ is defined as usually:

$$
M=i\left(q p-q^{*} p^{*}\right)=q_{1} p_{2}-q_{2} p_{1}=-(Q+1) .
$$

As a result, the choice of ordering of product of velocities in the quantum Lagrangian is an additional physical hypothesis, defining presence or lack of zero-point energy and charge.

\subsection{Negative norm problem for negative-frequency states and its solution}

$C$-conjugate states, considered in the previous section, are similar to ordinary states going backward in time and this representation is often more convenient for description of processes in relativistic theory. Here we will consider the problems of such representation and their solution.

In quantum mechanics the time reversal operation $t \rightarrow-t$ can be introduced in two forms. In the first one, accepted in the nonrelativistic theory, at the time reversal $T$ a sign of energy remains positive $H \rightarrow H$, but $T$ is described by anti-unitary operator with Hermite conjugation of matrix elements that is hidden change of the sign of energy.

In the second form, often used in the relativistic theory in combination with $\mathrm{CP}$ symmetry, $T$ is a part of 4-inversion $x^{\mu} \rightarrow-x^{\mu}(P T)$ at which all components of 4momentum change a sign: $p_{\mu} \rightarrow-p_{\mu}$, i.e. the sign of energy changes also and it is described by unitary operator. States with $-p_{\mu}$ evolve backward in time only and they describe antiparticles with $+p_{\mu}$, evolving forward in time only.

This treatment allows one to construct causal propagator of particles. Thus, an initial state of a particle with $-p_{\mu}$ represents a final state of its antiparticle with $+p_{\mu}$. This is widely using in particle physics crossing symmetry of processes when the changing of a sign of 4-momentum in a diagram or rotation of a line around a vertex with transfer into opposite light cone transforms particle to antiparticle (and contrary).

Further we will take this second form of the time reversal as a part of 4-inversion $P T$ naturally coinciding with $C P T$-symmetry in general and with the crossing symmetry in particular cases. Thus, at the time reversal $t \rightarrow-t$ it will be changed both a sign on frequency $\omega \rightarrow-\omega$ and a sign on energy $H \rightarrow-H$ which for the harmonic oscillator's quanta directly follows from the formula for energy.

For the harmonic oscillator the frequency appears in the form of products - with mass $(m \omega)$, with time $(\omega t)$ or quadratically, and only in the formula for energy enters linearly and determines a sign of energy. The expression for the harmonic oscillator ground state wave function for both signs of frequency

$$
\left|0_{ \pm}\right\rangle \rightarrow \psi_{0}\left(x_{ \pm}\right)=(m \omega / \pi)^{1 / 4} e^{-m \omega x_{ \pm}^{2} / 2}
$$


will remain real and finite at $x^{2} \rightarrow \infty$ only at joint change of signs of frequency and mass $\omega \rightarrow-\omega, m \rightarrow-m$, when their product does not change a sign: $m_{ \pm} \omega_{ \pm}=m \omega$, where $m>0, \omega>0$ and $m_{ \pm}= \pm m, \omega_{ \pm}= \pm \omega$. The wave functions will not vary if $\omega_{ \pm} \cdot( \pm t)=\omega t$, and the commutators of ladder operators

$$
\left[a_{ \pm}, a_{ \pm}^{*}\right]=1
$$

do not depend on the frequency and mass, so they will remain unchanged too.

At former approaches to treatment of states of the oscillator it has been implicitly suggested that a positive mass in negative-frequency states is positive. As the result, this led to the negative sign in the commutator $\left[a_{-}, a_{-}^{*}\right]=-1$ instead of (38) and then to the negative norm of such states. This assumption in practice is unphysical since the mass in the relativistic theory is, up to $c^{2}$, the energy in the rest frame $E_{0}=m c^{2}$.

Therefore if the signs of mass, frequency and energy are the same, commutators are positive as in (38), and the norm of the negative frequency state is positive. Thus, it makes consecutive the theory of the oscillator with both signs of frequency, the relations of which take the following form.

The Lagrangian and Hamiltonian of harmonic oscillators of two types differing by a sign of frequency $\omega_{ \pm}= \pm \omega, \omega \geq 0$, with the real (generalized) coordinates $x_{ \pm}$, masses $m_{ \pm}$and momenta $p_{ \pm}=m_{ \pm} \partial_{t} x_{ \pm}$, have the form:

$$
\begin{aligned}
& L_{ \pm}=\frac{m_{ \pm}}{2}\left[\left(\partial_{t} x_{ \pm}\right)^{2}-\omega^{2} x_{ \pm}^{2}\right], \\
& H_{ \pm}=\frac{1}{2 m_{ \pm}}\left[p_{ \pm}^{2}+(m \omega)^{2} x_{ \pm}^{2}\right]
\end{aligned}
$$

Their sign is defined by a sign of mass $m_{ \pm}$. Equations of motion $\partial_{t}^{2} x_{ \pm}+\omega^{2} x_{ \pm}=0$ lead to frequency decomposition of the coordinates and momenta:

$$
\begin{gathered}
x_{ \pm}=\frac{1}{\sqrt{2 m \omega}}\left(a_{ \pm} e^{-i \omega t}+a_{ \pm}^{*} e^{i \omega t}\right), \quad p_{ \pm}=\frac{-i m \omega}{\sqrt{2 m \omega}}\left(a_{ \pm} e^{-i \omega t}-a_{ \pm}^{*} e^{i \omega t}\right) . \\
a_{ \pm}=\frac{e^{i \omega t}}{\sqrt{2 m \omega}}\left(m \omega x_{ \pm}+i p_{ \pm}\right), \quad a_{ \pm}^{*}=\frac{e^{-i \omega t}}{\sqrt{2 m \omega}}\left(m \omega x_{ \pm}-i p_{ \pm}\right),
\end{gathered}
$$

At quantization commutators $i\left[p_{ \pm}, x_{ \pm}\right]=1$ lead to commutators (38) for ladder operators. Thus, the operators of opposite sign frequency states mutually commutate since they act on different state spaces. These states of both types of oscillators are defined by acting of corresponding ladder operators on their ground and excited states $\left|n_{ \pm}\right\rangle$:

$$
a_{ \pm}\left|n_{ \pm}\right\rangle=\sqrt{n_{ \pm}}\left|n_{ \pm}-1\right\rangle, \quad a_{ \pm}^{*}\left|n_{ \pm}\right\rangle=\sqrt{n_{ \pm}+1}\left|n_{ \pm}+1\right\rangle, \ldots
$$

where $n_{ \pm}=0,1,2, \ldots$ The Hamiltonian and number operator of quanta $N_{ \pm}$take a form:

$$
H_{ \pm}=\omega_{ \pm}\left(N_{ \pm}+1 / 2\right), \quad N_{ \pm}=a_{ \pm}^{*} a_{ \pm} .
$$

Thus, the standard theory of the harmonic oscillator extended with inserting of negative-frequency states, is self-consistent if we always take the same signs of mass and energy. The ground states are defined as lowest energy state for the positive-frequency sector and highest energy state for the negative-frequency sector. 
Let us redefine coordinates and momenta so that further the dimensional constant $m=\left|m_{ \pm}\right|$, a value of mass, will not figure in formulas:

$$
\tilde{x}_{ \pm}=\sqrt{m} x_{ \pm}, \quad \tilde{p}_{ \pm}=p_{ \pm} / \sqrt{m} .
$$

The Lagrangian, momenta and the Hamiltonian of the system then take the form:

$$
\begin{gathered}
L_{ \pm}= \pm \frac{1}{2}\left[\left(\partial_{t} \tilde{x}_{ \pm}\right)^{2}-\omega^{2} \tilde{x}_{ \pm}^{2}\right], \\
H_{ \pm}= \pm \frac{1}{2}\left(\tilde{p}_{ \pm}^{2}+\omega^{2} \tilde{x}_{ \pm}^{2}\right), \quad \tilde{p}_{ \pm}= \pm \partial_{t} \tilde{x}_{ \pm} .
\end{gathered}
$$

Notice the changing a sign of negative-frequency contributions. The commutators and equations of motion thus will not vary, and the frequency decomposition takes the form:

$$
\tilde{x}_{ \pm}=\frac{1}{\sqrt{2 \omega}}\left(a_{ \pm} e^{-i \omega_{ \pm} t}+a_{ \pm}^{*} e^{i \omega_{ \pm} t}\right), \quad \tilde{p}_{ \pm}=\frac{-i \omega}{\sqrt{2 \omega}}\left(a_{ \pm} e^{-i \omega_{ \pm} t}-a_{ \pm}^{*} e^{i \omega_{ \pm} t}\right)
$$

where the ladder operators $a_{ \pm}, a_{ \pm}^{*}$ remained unchanged, but expressed in the form:

$$
a_{ \pm}=\frac{e^{i \omega_{ \pm} t}}{\sqrt{2 \omega}}\left(\omega x_{ \pm}+i p_{ \pm}\right), \quad a_{ \pm}^{*}=\frac{e^{-i \omega_{ \pm} t}}{\sqrt{2 \omega}}\left(\omega x_{ \pm}-i p_{ \pm}\right) .
$$

The commutators (38) and norms of states are positive at both signs of frequency.

Above the harmonic oscillators with opposite signs of frequency (and masses) were considered separately. Let us consider a model including simultaneously the states of both sign of frequency. The negative-frequency state describes an oscillating antiparticle in terms of variables of a particle evolving inversely in time. Then the Lagrangian and Hamiltonian of the system look like:

$$
\begin{aligned}
L & =\frac{m_{+}}{2}\left[\left(\partial_{t} x_{+}\right)^{2}-\omega^{2} x_{+}^{2}\right]+\frac{m_{-}}{2}\left[\left(\partial_{t} x_{-}\right)^{2}-\omega_{-}^{2} x_{-}^{2}\right], \\
H & =\frac{1}{2 m_{+}}\left[p_{+}^{2}+(m \omega)^{2} x_{+}^{2}\right]+\frac{1}{2 m_{-}}\left[p_{-}^{2}+(m \omega)^{2} x_{-}^{2}\right] .
\end{aligned}
$$

At redefinition of variables (45)-(48) with excluding $m=\left|m_{ \pm}\right|$we have:

$$
\begin{gathered}
L=\frac{1}{2}\left[\left(\partial_{t} \tilde{x}_{+}\right)^{2}-\omega^{2} \tilde{x}_{+}^{2}\right]-\frac{1}{2}\left[\left(\partial_{t} \tilde{x}_{-}\right)^{2}-\omega^{2} \tilde{x}_{-}^{2}\right], \\
H=\frac{1}{2}\left(\tilde{p}_{+}^{2}+\omega^{2} \tilde{x}_{+}^{2}\right)-\frac{1}{2}\left(\tilde{p}_{-}^{2}+\omega^{2} \tilde{x}_{-}^{2}\right) .
\end{gathered}
$$

Then the Hamiltonian and the number operator take the form:

$$
H=\omega_{+}\left(N_{+}+1 / 2\right)+\omega_{-}\left(N_{-}+1 / 2\right) .
$$

Thus, at redefinition of variables (45)-(48) the mass parameter formally does not figure in formulas, but nevertheless the sign of mass remains and it changes a sign of negative-frequency contributions in the Lagrangian and Hamiltonian. This property allows us to introduce the negative-frequency modes into the field theories also, where field's "oscillators" appear without the mass parameter.

\subsection{Harmonic and magneto-harmonic rotators}

Above a planar harmonic oscillator was considered as analog of an oscillator with complex variables, while now we will study it from another point of view. Let a particle of mass $m$ moves in the harmonic potential $V=m \omega^{2} r^{2} / 2$ on a plane with the Cartesian $(x, y)$ or polar $(r, \theta)$ coordinates. 
If angular momentum $M= \pm m r^{2} \dot{\theta}$ is absent $(M=0)$, the particle fluctuates along the line passing through the center, and particle's kinetic energy is reduced to the pure radial contribution $m \dot{r}^{2} / 2$. By turning the coordinate system the radius vector can be directed along $x$ axis and the planar oscillator passes to the linear harmonic oscillator.

At nonzero angular momentum $M \neq 0$, which conserves, the centrifugal energy $m r^{2} \dot{\theta}^{2} / 2=M^{2} / 2 m r^{2}$ together with harmonic potential forms the effective potential. It is singular at $r \rightarrow 0$ and the particle does not pass through the center, but rotates around it on an orbit. Such oscillator with rotational mode is not reduced to one linear oscillator and it has been represented simply as two linear oscillators for both axes $(x, y)$.

But this simplification is incorrect in a case when the number of dynamic degrees of freedom is less. If radial speed vanishes $\dot{r}=0$, the particle rotates around the center on a circular orbit and its kinetic energy reduces to the centrifugal energy $M^{2} / 2 m r^{2}$. In system again only one degree of freedom is dynamic, but now it becomes $\theta$, while the radial coordinate $r$ is a fixed parameter determining by frequency and total energy. Such purely planar oscillator is the harmonic rotator [4], the Lagrangian and Hamiltonian of which are:

$$
\begin{gathered}
L_{0}=\frac{1}{2} m r^{2} \dot{\theta}^{2}-\frac{1}{2} m \omega^{2} r^{2}=r^{2} \frac{m}{2}\left(\dot{\theta}^{2}-\omega^{2}\right), \\
H_{0}=M \dot{\theta}-L_{0}=\frac{1}{2 m}\left(\frac{M^{2}}{r^{2}}+m^{2} \omega^{2} r^{2}\right)=\left.M \omega\right|_{\dot{\theta}= \pm \omega} .
\end{gathered}
$$

Here $r^{2}$ became Lagrange multiplier, and Lagrangian is reduced to constraint $\varphi \sim \dot{\theta}^{2}-\omega^{2}$. The Schrödinger equation for the harmonic rotator with this constraint is:

$$
i \hbar \partial_{t} \psi=\frac{1}{2 m}\left(\frac{M^{2}}{r^{2}}+m^{2} \omega^{2} r^{2}\right) \psi=\left.M \omega \psi\right|_{\dot{\theta}= \pm \omega} .
$$

Energy of the harmonic rotator is proportional to $\omega$, the frequency of circular rotation which is constant, and to $M_{n_{\theta}}= \pm m r_{n_{\theta}}^{2} \omega=n_{\theta} \hbar$, the angular momentum, which conserves and quantized $n_{\theta}=0, \pm 1, \ldots$ The energy spectrum is equidistant, linear on rotation frequency, twice degenerated and starts from zero:

$$
E_{n, s}=\hbar \omega\left|n_{\theta}\right| \text {. }
$$

The harmonic rotator can be obtained from the planar harmonic oscillator also by imposing of a constraint vanishing the radial momentum [4]. At this approach the radial momentum does not contribute to the energy and after quantization there are no radial modes and their zero-point energy.

A standard physical realization of the harmonic rotator is the motion of a charged particle in a constant uniform magnetic field $H_{z}$. In the classical theory the particle moves on the plane $(x, y)$ along circular orbits and the magnetic field acts on this plane of rotation as a harmonic potential. Thus, the equation of motion for radial degree of freedom on the plane $r=\left(x^{2}+y^{2}\right)^{1 / 2}$ is trivial $\dot{r}=0$ and leads to vanishing of the radial momentum. Quantization with conservation of radial coordinate as a constrained dynamic degree of freedom gives the zero-point energy for radial modes and further leads to violation of $C$ symmetry of the system [4]. 
Therefore if at quantization we want to keep exact $C$-symmetry of the system, we should take the contributions of purely rotational modes only, not having zero-point energy. The Lagrangian of such magneto-harmonic rotator can be chosen in two forms or we start from the standard classical Lagrangian

$$
L_{\perp}=\frac{m}{2} \dot{r}^{2}+r^{2} \frac{m}{2}\left[\left(\dot{\theta}+\omega_{ \pm}\right)^{2}-\omega_{ \pm}^{2}\right] .
$$

by introducing the $C$-symmetry requirements, or we choose initially the kinetic term without radial speed:

$$
L_{0}=r^{2} \frac{m}{2}\left[\left(\dot{\theta}+\omega_{ \pm}\right)^{2}-\omega_{ \pm}^{2}\right] .
$$

Lagrangian (60) differs from (55) only by the shift of angular speed: $\dot{\theta} \rightarrow \dot{\theta}+\omega_{ \pm}$. Then only the rotational modes of the harmonic rotator contribute to the energy spectrum:

$$
E_{n_{\theta}}=\omega\left(\left|n_{\theta}\right|-n_{\theta}\right)=\left\{\begin{array}{cc}
\omega_{H}\left|n_{\theta}\right|, & n_{\theta}<0, \\
0, & n_{\theta}>0 .
\end{array}\right.
$$

Cyclotron frequency $\omega_{H}=e\left|H_{z}\right| / m_{0} c$ is twice more than a frequency of the harmonic rotator: $\omega_{H}=2 \omega_{ \pm}$, and radii of levels in $\sqrt{2}$ times is less. Thus, in the magneto-harmonic rotator with the Lagrangian (60) the spectrum starts from zero. Standard choice of the Lagrangian (59), giving the zero-point energy, is useful at introduction of complex variables and we will consider it below too.

In two given physical examples contributions of rotational modes were separated from contributions of radial modes that allowed us to choose a model Lagrangian without radial kinetic term. In general case when such separation is not obvious, the problem consists in the restriction by the contribution of rotational modes only. For complex variables $C$-symmetry of states will help in reaching that.

At quantization of the magneto-harmonic rotator in complex variables on the rotation plane the complex conjugate velocities do not commutate: $\left[\dot{q}, \dot{q}^{*}\right]=2 \omega / \mathrm{m}$. Therefore, there are two types of Lagrangian with different ordering of products of speeds which in the classical case pass into the standard classical Lagrangian and, thus, were considered as equivalent ones:

$$
\begin{aligned}
& L_{\perp}=m \dot{q} \dot{q}^{*}-m \omega \cdot i\left(q^{*} \dot{q}-\dot{q}^{*} q\right) . \\
& L_{0}=m \dot{q}^{*} \dot{q}-m \omega \cdot i\left(q^{*} \dot{q}-\dot{q}^{*} q\right),
\end{aligned}
$$

By taking into account this property, the Lagrangians (59)-(60) we can rewrite as:

$$
\begin{gathered}
L_{\perp}=m\left[\left(D_{t} q\right)\left(D_{t} q\right)^{*}-\omega^{2} q^{*} q\right]-\omega, \\
L_{0}=m\left[\left(D_{t} q\right)^{*}\left(D_{t} q\right)-\omega^{2} q^{*} q\right] .
\end{gathered}
$$

where a "covariant" time derivative is $D_{t}=\partial_{t}+i \omega$. Unlike velocities, $D_{t} q$ and $\left(D_{t} q\right)^{*}$ which are momenta up to a coefficient (mass), commutate. From (64)-(65) we have:

$$
\begin{gathered}
H_{\perp}=\frac{1}{m}\left(p+i m \omega q^{*}\right)\left(p^{*}-i m \omega q\right)+\omega=2 \omega a^{*} a+\omega=\omega_{H}(Q+1 / 2), \\
H_{0}=\frac{1}{m}\left(p+i m \omega q^{*}\right)\left(p^{*}-i m \omega q\right)=2 \omega a^{*} a=\omega_{H} Q,
\end{gathered}
$$

where a «chiral charge» operator $Q$ is defined in (4). 
Thus, $L_{\perp}$ gives $H_{\perp}$ with the standard ordering and the zero-point energy $E_{(0)}=\omega_{H} / 2$, which in the magnetic field appears as chaotic motions along arcs. At the same time $L_{0}$ leads to $H_{0}$ without zero-point energy and, therefore, the such system also is the magneto-harmonic rotator.

\subsection{The rotating chains of harmonic rotators}

The spectrum of a harmonic rotator equidistant, degenerate and does not contain the zero-point energy that takes place for quantized relativistic fields with gauge and $C$ symmetry. Since the fields are similar to the continuous limit of chains of rotators, it is natural to consider a chain of rotators, particularly in terms of complex variables too.

Let there is a set of $N$ identical harmonic rotators, each built as a system of two elastically connected particles $A_{i}$ and $B_{i}$, rotating on planes $\left(x, y, z_{i}\right)$, where $i=1, \ldots, N$ , and let centers of mass of these rotators are located by step $d$ along a common rotation axis $z$. At identical angular speed $\omega_{0}$ and identical starting conditions the total set of rotators rotates coherently and, because of the angular momentum $\left(M_{ \pm}\right)$conservation, each of them remains on own rotation plane. Energy of such system is:

$$
E_{N}=N_{+} M_{+} \omega_{0+}+N_{-} M_{-} \omega_{0-}+E_{0},
$$

Let us join by means of elastic force $\kappa\left(r_{i+1}-r_{i}\right)$ each of two particles of each of rotators to similar particles of two neighbor rotators ( $A_{i}$ with $A_{i \pm 1}$, and $B_{i}$ with $B_{i \pm 1}$ ), constructing a double one-dimensional chains of $2 \mathrm{~N}$ particles. In the continuous limit this double chain forms two massive strings joined by an elastic thin film. The rotation frequency of each of the harmonic rotators $\omega_{0}=\sqrt{\kappa / m}$ is constant and its connections with neighbors do not change it. They only lead to an angular momentum changing at an additional tension on a rotation plane, thus because of the angular momentum conservation and weakness of connections with neighbors, each rotator remains on own rotation plane.

Let now an external force adds to one of rotators in the chain an additional angular momentum and corresponding energy. This angular momentum will be transferred on the chain, while all chain will synchronously rotate as before, only small extra tension will move over the double chain. At a periodical perturbation a transverse wave of tensions of rotators will propagate in the chain. The length of this wave $\lambda=l^{\prime} d$ is determined by a number $l^{\prime}$ of the rotators covered by wave's period and corresponding wave vector $\mathbf{k}_{z}=2 \pi \mathbf{n}_{z} / \lambda$, directed along $z$, represents a momentum of wave's quanta. There with $\mathbf{k}_{z}$ it is related a "longitudinal" frequency of the propagating wave, defined as a product of $k_{z}$ on wave's velocity $\omega_{z}=v_{z} k_{z}$. Wave's crests of two opposite ends of rotators on a rotation plane $(x, y)$ rotate with the common rotation frequency $\omega_{0}$, move along $z$ with velocity $v_{z}$ and their trajectory in space form two helicoid curves around $z$ axis.

In quantum theory where each a rotator has a quantized angular momentum and corresponding energy levels, the wave's passing consists in transition to the next level and going back, so there is a propagation of a quasi-particle at adding of the angular momentum quantum and holes at its removing. If to the same rotator on the one hand chain the quasiparticle arrives, and on the other hand simultaneously a hole arrives, the angular momentum does not change and thus there is a recombination of these perturbations. 
In a system of isolated rotators, also located along the same common rotation axis $\left(x_{i}, y_{i}\right)$ and coherently rotating, there is a rotational symmetry $O(2)$ in the space of two degrees of freedom with a conserved angular momentum. In a complex space $\left(q_{n}^{*}, q_{n}\right)$ a rotational symmetry corresponds to the group $U(1)$ with a conserved charge.

When rotators form the elastically bounded double chain, the connections of rotators with each other lead to a new effect of the topological nature - the system transfers into itself not at rotation one of rotators on angle $2 \pi$, as for the isolated rotator, but at its rotation on $4 \pi$, and thus a symmetry group at rotations becomes $S U(2)$. The reason is that, at rotation on $4 \pi$ one of the rotators, the chain can be untangled (by Dirac's method) and it is returned into a state before rotation without a changing of boundaries of the chain and without a changing of orientation of the turned rotator. Thus, in the chain of the bounded rotators there appear naturally quanta in the spinor representation of the rotation group.

The Lagrangian of a connected chain of harmonic rotators can be written as:

$$
L=\sum_{n=1}^{N}\left[m \dot{q}_{n}^{*} \dot{q}_{n}-k\left(q_{n+1}^{*}-q_{n}^{*}\right)\left(q_{n+1}-q_{n}\right)-m \omega_{0}^{2} q_{n}^{*} q_{n}\right]
$$

The canonical momenta $p_{n}=m \dot{q}_{n}^{*}, \quad p_{n}^{*}=m \dot{q}_{n}$ give the Hamiltonian and charge:

$$
\begin{gathered}
H=\sum_{n=1}^{N}\left[m^{-1} p_{n}^{2} p_{n}^{* 2}+\kappa\left(q_{n+1}^{*}-q_{n}^{*}\right)\left(q_{n+1}-q_{n}\right)+m \omega_{0}^{2} q_{n}^{*} q_{n}\right] . \\
Q=i \sum_{n=1}^{N}\left(q_{n}^{*} p_{n}^{*}-p_{n} q_{n}\right),
\end{gathered}
$$

The equations of motion following from this Hamiltonian are

$$
\ddot{q}_{n}+\omega_{0}^{2}\left(2 q_{n}-q_{n+1}-q_{n-1}\right)+\omega_{0}^{2} q_{n}=0,
$$

which in the continuous limit gives the wave equation

$$
\partial_{t}^{2} q-\mathrm{v}^{2} \partial_{r}^{2} q+\omega_{0}^{2} q=0
$$

with the speed of wave $\mathrm{v}=\omega_{0} d$. These equations describe the transverse waves along two connected by an elastic film and rotating strings. It is possible to enter periodic boundary conditions and for a large number of rotators:

$$
q_{1}(t)=q_{N+1}(t), q_{1}^{*}(t)=q_{N+1}^{*}(t) .
$$

Since there is a coherent rotation of all of rotators with common frequency $\omega_{0}$, the rotation energy of each of rotators is expresses through the angular momentum which now contains the contribution of frequency of the wave also. Thus, as before it is possible to present chain of rotators as a set of independent rotators (normal modes), but only with the frequencies $\omega_{k}$, following, as for a chain of linear oscillators, from the wave equation. Thus, it is possible to represent the waves on the chain of rotators as a series of independent normal modes of frequencies $\omega_{k}$, following from the wave equations (72):

$$
\begin{gathered}
\ddot{a}_{k}(t)+\omega_{k}^{2} a_{k}(t)=0, \\
\omega_{k}^{2}=\omega_{0}^{2}\left(2-e^{i k d}-e^{-i k d}\right)+\omega_{0}^{2}=\mathrm{v}^{2} k^{2}+\omega_{0}^{2}, \\
\mathrm{v}^{2} k^{2}=2 \omega_{0}^{2}(1-\cos k d) \approx k^{2} \omega_{0}^{2} d^{2} .
\end{gathered}
$$

This gives following frequency decomposition of the generalized coordinates: 


$$
\begin{aligned}
& q_{n}(t)=\sum_{k} \frac{1}{\sqrt{2 m N \omega_{k}}}\left(a_{k} e^{-i \omega_{k} t+i k n d}+\beta_{k}^{*} e^{i \omega_{k} t-i k n d}\right), \\
& q_{n}^{*}(t)=\sum_{k} \frac{1}{\sqrt{2 m N \omega_{k}}}\left(a_{k}^{*} e^{i \omega_{k} t-i k n d}+\beta_{k} e^{-i \omega_{k} t+i k n d}\right),
\end{aligned}
$$

( $k=2 \pi l^{\prime} / N d, l^{\prime}$-integers). This decomposition corresponds to a case when at rotations of the doubled chain of the bounded rotators there is a propagation of transverse waves along $z$ axis with the wave numbers $k$ and frequencies $\omega_{k}$. Notice that the wavelength in the chain sufficiently exceeds distances between the rotators. The angular momentum, energy and the momentum of these waves are given by the expressions:

$$
\begin{aligned}
& H=\sum_{k} \omega_{k} \cdot Q_{k}=\sum_{k} \omega_{k}\left(a_{k}^{*} a_{k}+\beta_{k} \beta_{k}^{*}\right), \\
& Q=\sum_{k} Q_{k}=\sum_{k}\left(a_{k}^{*} a_{k}-\beta_{k} \beta_{k}^{*}\right), \\
& P=\sum_{k} k\left(a_{k}^{*} a_{k}+\beta_{k} \beta_{k}^{*}\right) .
\end{aligned}
$$

Ladder operators with nonzero commutators:

$$
\left[a_{k}, a_{k^{\prime}}^{*}\right]=\delta_{k k^{\prime}}, \quad\left[\beta_{k}, \beta_{k}^{*}\right]=\delta_{k k^{\prime}}
$$

give for the conserving observables of the normal modes:

$$
\begin{aligned}
& H_{k}=\omega_{k}\left(a_{k}^{*} a_{k}+\beta_{k} \beta_{k}^{*}\right), \\
& Q_{k}=a_{k}^{*} a_{k}-\beta_{k} \beta_{k}^{*} .
\end{aligned}
$$

Introducing corresponding $C$-conjugate ladder operators:

$$
b_{k} \equiv C a_{k} C^{-1}, \quad b_{k}^{*} \equiv C a_{k}^{*} C^{-1}, \quad \alpha_{k} \equiv C \beta_{k} C^{-1}, \quad \alpha_{k}^{*} \equiv C \beta_{k}^{*} C^{-1},
$$

for the $C$-conjugate observables we obtain the expressions:

$$
H^{c}=\sum_{k} H_{k}^{c}=\sum_{k} \omega_{k}\left(b_{k}^{*} b_{k}+\alpha_{k} \alpha_{k}^{*}\right), \quad Q^{c}=\sum_{k} Q_{k}^{c}=\sum_{k}\left(b_{k}^{*} b_{k}-\alpha_{k} \alpha_{k}^{*}\right) .
$$

The difference of a wave on the chain of connected rotators from the common rotation by a constant frequency is in summation on normal modes with different wave frequencies. Since the modes with different frequencies are independent, each mode also is $C$-symmetric and the symmetry requirements relate the $C$-conjugate operators of observables in each mode:

$$
H_{k}^{c}=H_{k}, Q_{k}^{c}=-Q_{k},
$$

from which, by means of earlier shown procedure, follow the relations:

$$
\alpha_{k} \alpha_{k}^{*}=a_{k}^{*} a_{k}, \quad \beta_{k} \beta_{k}^{*}=b_{k}^{*} b_{k} \text {. }
$$

By substituting them into (80) or (82), the observables can be expressed in terms of the operators of the mutually $C$-conjugate quanta:

$$
\begin{aligned}
& H=\sum_{k} H_{k}=\sum_{k} \omega_{k}\left(a_{k}^{*} a_{k}+b_{k}^{*} b_{k}\right), \\
& Q=\sum_{k} Q_{k}=\sum_{k}\left(a_{k}^{*} a_{k}-b_{k}^{*} b_{k}\right), \\
& P=\sum_{k} P_{k}=\sum_{k} k\left(a_{k}^{*} a_{k}+b_{k}^{*} b_{k}\right) .
\end{aligned}
$$

Thus, in the linear chain of harmonic rotators with $C$-symmetry a zero-point energy and a zero-point charge do not arise. 


\section{Rotatory quantization of $C$-symmetric fields}

\subsection{Experimental bases for rotatory quantization}

In this section the rotational quantization based on the harmonic rotator will applied to fields where the frequency of quanta will mean the frequency of rotation of field vectors. Charges and spin will appear then naturally, and the zero-point energy and the zero-point charge of vacuum will not arise due to $C$-symmetry. The absence of the zero-point fluctuations of fields can be checked in many experiments and thus we begin with the al bases for the rotational quantization of fields.

In particle physics the well known observed effects - the Lamb shift, the anomalous magnetic moment and the Casimir effect - contain separate contributions not only from quantum fluctuations of fields of real sources, but, if they are exist, from the zero-point fluctuations of fields too. Really, with the zero-point energy $H_{0}^{(0)}$ of electromagnetic field would be connected vacuum fields $\mathbf{E}_{(0)}, \mathbf{B}_{(0)}$ :

$$
H_{0}^{(0)}=2 \int d^{3} k \frac{1}{2} \omega_{k}=\int d^{3} x \frac{1}{2}\left(\mathbf{E}_{(0)}^{2}+\mathbf{H}_{(0)}^{2}\right)
$$

and in a total energy there would be inevitably a contribution from energy of interaction of charges with this vacuum field $H_{I}^{(0)}=\mathbf{j} \cdot \mathbf{A}_{(0)}$, where $\mathbf{A}_{(0)}$ - potential corresponding $\mathbf{E}_{(0)}, \mathbf{B}_{(0)}$ [4]. But this energy of interaction $H_{I}^{(0)}$ enters into the Hamiltonian additively with the interaction energy $H_{I}^{(r)}=\mathbf{j} \cdot \mathbf{A}_{(r)}$ of the same particles with fields $\mathbf{A}_{(r)}$ of real sources, so both types of contributions should be summed. Since both contributions are the same order (because of they often confused), energy shifts would be doubled:

$$
\Delta E_{I}=\Delta E_{I}^{(0)}+\Delta E_{I}^{r} \approx 2 \Delta E_{I}^{r} \text {. }
$$

The observation the existence only a single contribution

$$
\Delta E_{I}^{\exp }=\Delta E_{I}^{r},
$$

which evidently is the contribution $\Delta E_{I}^{r}$ of real sources, while the predicted contribution of the hypothetical vacuum fields remain invisible in each experiment. Thus, the existing experiments contradict to the vibrational treatment of fluctuations of fields and confirm the rotational quantization.

In this regard let us consider some details of comparison of predictions of both treatments. In the Hamiltonians of the oscillatory $\left(H_{o s c}\right)$ and rotatory $\left(H_{r o t}\right)$ quantizations compare contributions of separate terms to observable effects. An energy of free electrons and photons $H_{0}^{(r)}$ and energy of their interactions $H_{I}^{(r)}$ in both cases are identical. Only distinction is that $H_{\text {osc }}$ contains the zero-point vacuum energy $H_{0}^{(0)}$ in the free Hamiltonian and the interaction energy of charges with fluctuating vacuum fields $H_{I}^{(0)}$, while at the rotatory treatment of $C$-symmetric systems the zero-point energy is absent:

$$
\begin{aligned}
& H_{o s c}=\left(H_{0}^{(r)}+H_{I}^{(r)}\right)+\left[H_{0}^{(0)}+H_{I}^{(0)}\right], \\
& H_{r o t}=\left(H_{0}^{(r)}+H_{I}^{(r)}\right) .
\end{aligned}
$$

Terms in round parentheses in (89) and (90) are the same in both Hamiltonians since relate to the real sources. They lead to the diagram techniques, the loop contributions of which completely explain the observed values of the Lamb shift and the anomalous 
magnetic moments as effects from $H_{I}^{(r)}$. Thus, rotational quantization without zero-point contributions is in agreement with these experiments.

At the same time, in vibrational approach in addition to these contributions in there will be also contributions to $H_{o s c}$ from $H_{I}^{(0)}$ which, as we know, are the same order as the loop contributions from $H_{I}^{(r)}$. The sum these two independent contributions give almost twice more value (87), than observing one (88), so in fact the vibrational approach to fields is rejected by these experiments.

In case of Casimir effect the terms in parentheses in (90) and (89) contribute through a fluctuating radiation field of crystal's atoms. As it is well known, the theory of Van der Waals forces, improved by other methods, successfully describes the Casimir effect, including temperature dependences and properties of materials, through radiation of real sources, atoms, at their zero-point vibrations (i.e. vibrations at crystal's zero temperature (!)). Since the contributions of purely vacuum fields from $H_{I}^{(0)}$ are the same order and must be added to the contribution from the radiation of atoms $\left(H_{I}^{(r)}\right)$, in this case the oscillatory approach gives almost twice larger value for the Casimir effect than observing one and in fact is rejected by these experiments. Rotatory quantization only with contributions of fields of atoms again is in agreement with these experiments.

The observations also show a practically vanishing (from the particle physics scales) value for the cosmological constant. This fact also cannot be explained in the vibrational quantization, and in the vibrational quantization all known attempts to exclude this energy have required more and more unrealistic hypotheses. At the same time rotational quantization directly and without hypotheses explains the lack of sensible contribution to the cosmological constant from the vacuum of known fundamental fields.

\subsection{Rotatory quantization of the photon field}

At quantization of electromagnetic field the circularly polarized waves with two helicity projections form pure states of the photon field at which the Hamiltonian and helicity operator $\Lambda=\Lambda^{0}$, the time component of divergence of the total angular momentum $\Lambda^{\mu}=\partial_{v} J^{\mu v}$, are diagonal. In the flat wave the helicity is a projection of the spin $\mathbf{S}$ to the momentum direction: $\Lambda=\mathbf{S} \cdot \mathbf{k} /|\mathbf{k}|$.

Photons of opposite helicity are emitted by a rotating dipole and they behave as two states of opposite sign chiral charges. At dipole transitions between rotational levels $\Delta n= \pm 1$ energies of emitted photons are identical and equal to $\Delta E=\hbar \omega$, where $\omega$ is a cyclic rotation frequency. Thus, photons are similar to quanta of a harmonic rotator. Symmetry between photons of a different helicity thus is analog of $C$-symmetry that leads to the lack of the zero-point energy, so that analogy by the harmonic rotator is exact concerning field's vacuum too.

For our purposes it is enough to deal with physical degrees of freedom of the photon field in a convenient inertial frame. The revealed physical properties - the rotational nature of frequency and lack of zero-point energy - then will remain in other gauges and inertial frames too due to the invariance of properties of vacuum. Thus, we will direct an axis of spatial coordinates $x^{3}$ along photon's momentum $\mathbf{k}=\left(0,0, k^{3}\right)$ and choose the gauge $A_{3}=0$, having left only transverse physical components of the field vector $A_{1}, A_{2}$. By forming from latters a complex field operator $A$ :

$$
A(x)=\left(A_{1}+i A_{2}\right) / \sqrt{2}, \quad A^{*}(x)=\left(A_{1}-i A_{2}\right) / \sqrt{2},
$$


we can write the Lagrangian of the photon field as:

$$
L=\int d^{3} x \partial_{\mu} A^{*} \cdot \partial^{\mu} A \text {. }
$$

The Lagrangian is symmetric under the global phase transformations:

$$
A^{\prime}=e^{i \theta \Lambda} A, \quad A^{* \prime}=e^{-i \theta \Lambda} A^{*}
$$

and here corresponding conserving «chiral charge» $\Lambda$ is the helicity. The free Hamiltonian and the helicity operator are:

$$
\begin{aligned}
& H=\int d^{3} x\left(\pi^{*} \pi+\nabla A^{*} \nabla A\right), \\
& \Lambda=i \int d^{3} x\left(A^{*} \pi^{*}-\pi A\right)
\end{aligned}
$$

where $\pi(x)=\partial_{t} A^{*}, \pi^{*}(x)=\partial_{t} A$. Two operators $\mathrm{H}$ and $\Lambda$ become diagonal only for states of photons with circular polarization when the field vector is constant in modulo and rotates around the momentum direction with a constant angular speed. Therefore in the frequency decomposition of fields and sources the frequency means an angular speed of the rotating field vectors. At quantization the rotating source emits or absorbs photons carrying away or getting a quantum of angular momentum. As we see, the photon field in the given gauge and frame formally is like a complex scalar field represented as a set of quanta in chains of harmonic rotators.

Field equations and simultaneous commutators of fields look like:

$$
\begin{gathered}
\partial_{\mu} \partial^{\mu} A=0, \quad \partial_{\mu} \partial^{\mu} A^{*}=0 . \\
i\left[\pi(\mathbf{x}, t), A\left(\mathbf{x}^{\prime}, t\right)\right]=\delta_{x x^{\prime}}^{3}, \quad i\left[\pi^{*}(\mathbf{x}, t), A^{*}\left(\mathbf{x}^{\prime}, t\right)\right]=\delta_{x x^{\prime}}^{3}
\end{gathered}
$$

Let's we decompose the field on normal modes of rotational waves:

$$
\begin{gathered}
A(x)=\sum_{k}\left(a_{k} e^{-i k x}+\beta_{k}^{*} e^{i k x}\right), \quad A^{*}(x)=\sum_{k}\left(a_{k}^{*} e^{i k x}+\beta_{k} e^{-i k x}\right), \\
\pi(x)=i \sum_{k} \omega_{k}\left(a_{k}^{*} e^{i k x}-\beta_{k} e^{-i k x}\right), \quad \pi^{*}(x)=-i \sum_{k} \omega_{k}\left(a_{k} e^{-i k x}-\beta_{k}^{*} e^{i k x}\right) .
\end{gathered}
$$

where $k_{0}=\omega_{\mathbf{k}}, \omega_{\mathbf{k}}=|\mathbf{k}|$ and the sums are defined as $\sum_{k}=\int d^{3} k\left[(2 \pi)^{3} 2 \omega_{k}\right]^{-1 / 2}$. Here $a_{k}, a_{k}^{*}$ are ladder operators of photons of helicity $\Lambda=+1$ and $\beta_{k}, \beta_{k}^{*}$ quanta of $\Lambda=-1$ , but through $C$-symmetry conditions. Commutators of fields give us:

$$
\left[a_{k}, a_{k^{\prime}}^{*}\right]=\delta_{k^{\prime}}^{3},\left[\beta_{k}, \beta_{k^{\prime}}^{*}\right]=\delta_{k^{\prime}}^{3}
$$

By taking into account (97)-(98), the Hamiltonian and helicity (94) take the form:

$$
\begin{aligned}
& H=\int d^{3} k H_{k}=\int d^{3} k\left(\Lambda_{k}^{(a)}-\Lambda_{k}^{(\beta)}\right) \omega_{k}=\int d^{3} k\left(a_{k}^{*} a_{k}+\beta_{k} \beta_{k}^{*}\right) \omega_{k}, \\
& \Lambda=\int d^{3} k \Lambda_{k}=\int d^{3} k\left(\Lambda_{k}^{(a)}+\Lambda_{k}^{(\beta)}\right)=\int d^{3} k\left(a_{k}^{*} a_{k}-\beta_{k} \beta_{k}^{*}\right) .
\end{aligned}
$$

At $C$-conjugation, in our case changing a sign of «chiral charge», the helicity, the right-rotating photon transforms to the left-rotating photon and vice versa. Here the $C$ symmetry conditions take a form:

$$
H_{k}^{c} \equiv C H_{k} C^{-1}=H_{k}, \quad \Lambda_{k}^{c} \equiv C \Lambda_{k} C^{-1}=-\Lambda_{k} .
$$

The operators $a_{k}, a_{k^{\prime}}^{*}$ will be $C$-conjugate, being transformed to $b_{k}, b_{k^{\prime}}^{*}$ the annihilation and creation operators of photons of helicity $\Lambda=-1$ and similar for $\beta_{k}, \beta_{k}^{*}$ : 


$$
\begin{array}{lll}
C a_{k} C^{-1}=b_{k}, & C a_{k}^{*} C^{-1}=b_{k}^{*}, & {\left[b_{k}, b_{k^{\prime}}^{*}\right]=\delta_{k k^{\prime}}^{3},} \\
C \beta_{k} C^{-1}=\alpha_{k}, & C \beta_{k}^{*} C^{-1}=\alpha_{k}^{*}, & {\left[\alpha_{k}, \alpha_{k^{\prime}}^{*}\right]=\delta_{k k^{\prime}}^{3},}
\end{array}
$$

They determine the frequency decomposition of $C$-conjugate field functions:

$$
A^{c}(x)=\sum_{k}\left(b_{k} e^{-i k x}+\alpha_{k}^{*} e^{i k x}\right), \quad A^{c^{*}}(x)=\sum_{k}\left(b_{k}^{*} e^{i k x}+\alpha_{k} e^{-i k x}\right) .
$$

The expressions for the $C$-conjugate helicity and Hamiltonian take a form:

$$
\begin{aligned}
& H^{c}=\int d^{3} k H_{k}^{c}=\int d^{3} k\left(\Lambda_{k}^{(b)}-\Lambda_{k}^{(\alpha)}\right) \omega_{k}=\int d^{3} k\left(b_{k}^{*} b_{k}+\alpha_{k} \alpha_{k}^{*}\right) \omega_{k}, \\
& \Lambda^{c}=\int d^{3} k \Lambda_{k}^{c}=\int d^{3} k\left(\Lambda_{k}^{(b)}+\Lambda_{k}^{(\alpha)}\right)=\int d^{3} k\left(b_{k}^{*} b_{k}-\alpha_{k} \alpha_{k}^{*}\right) .
\end{aligned}
$$

Now we write down $C$-symmetry conditions for field modes in details:

$$
\begin{aligned}
& H_{k}=\omega_{k}\left(\Lambda_{k}^{(a)}-\Lambda_{k}^{(\beta)}\right)=\omega_{k}\left(\Lambda_{k}^{(b)}-\Lambda_{k}^{(\alpha)}\right)=H_{k}^{c}, \\
& \Lambda_{k}=\Lambda_{k}^{(a)}+\Lambda_{k}^{(\beta)}=-\left(\Lambda_{k}^{(b)}+\Lambda_{k}^{(\alpha)}\right)=-\Lambda_{k}^{c},
\end{aligned}
$$

which give operator relations:

$$
\begin{aligned}
& \Lambda_{k}^{(a)}-\Lambda_{k}^{(\beta)}=\Lambda_{k}^{(b)}-\Lambda_{k}^{(\alpha)}, \\
& \Lambda_{k}^{(a)}+\Lambda_{k}^{(\beta)}=-\Lambda_{k}^{(b)}-\Lambda_{k}^{(\alpha)} .
\end{aligned}
$$

By adding and subtracting these relations, we obtain:

or in the explicit form:

$$
\Lambda_{k}^{(\beta)}=-\Lambda_{k}^{(b)}, \quad \Lambda_{k}^{(\alpha) p}=-\Lambda_{k}^{(a)} .
$$

$$
\beta_{k} \beta_{k}^{*}=b_{k}^{*} b_{k}, \quad \alpha_{k} \alpha_{k}^{*}=a_{k}^{*} a_{k} .
$$

After substituting in (100) and (104), we obtain final expressions for the observables:

$$
\begin{gathered}
H=H^{c}=\int d^{3} k\left(\Lambda_{k}^{(a)}-\Lambda_{k}^{(b)}\right) \omega_{k}=\int d^{3} k\left(a_{k}^{*} a_{k}+b_{k}^{*} b_{k}\right) \omega_{k} . \\
\Lambda=-\Lambda^{c}=\int d^{3} k\left(\Lambda_{k}^{(a)}+\Lambda_{k}^{(b)}\right)=\int d^{3} k\left(a_{k}^{*} a_{k}-b_{k}^{*} b_{k}\right) .
\end{gathered}
$$

Thus, the observable are expressed through helicity operators $\Lambda_{k}^{(a)}$ and $\Lambda_{k}^{(b)}$ for photons of opposite rotation direction. at $n_{a}=0, n_{b}=0$ the annihilation operators define the vacuum: $a_{k}|0\rangle=0, b_{k}|0\rangle=0$, which does not contain a zero-point energy. The helicity of the vacuum also is equal to zero.

Thus, quantization of the circularly polarized photon field is related by quantization of normal modes of the field as harmonic rotators, and in the ground state does not contain the zero-point energy. Because of invariance of properties of vacuum the fact of lack of the zero-point energy of vacuum, established in one a frame of reference and in one a gauge, is valid for all frames of reference and all gauges.

The above presented formalism of quantization of the photon field in the source's rest frame can be generalized to arbitrary inertial frames and presented in a relativisticallycovariant form, preserving the $\mathrm{C}$-symmetry, by means of tetrad vectors:

$$
\begin{aligned}
& A_{1}=e_{1}^{\mu} A_{\mu}, \quad A_{2}=e_{2}^{\mu} A_{\mu}, \quad e_{0}^{\mu} A_{\mu}=e_{3}^{\mu} A_{\mu}=0, \\
& A(x)=\frac{1}{\sqrt{2}}\left(e_{1}^{\mu}+i e_{2}^{\mu}\right) A_{\mu}=e_{+}^{\mu} A_{\mu}, \quad A^{*}(x)=\frac{1}{\sqrt{2}}\left(e_{1}^{\mu}-i e_{2}^{\mu}\right) A_{\mu}=e_{-}^{\mu} A_{\mu} .
\end{aligned}
$$

Then, the photon field, having two transverse components $\left(0, A_{1}, A_{2}, 0\right)$ in the rest frame system of the source, after the Lorentz transformation to other frames of reference in 
general has all four components $A_{\mu}=e_{\mu}^{1} A_{1}+e_{\mu}^{2} A_{2}$. The longitudinal component appears simply due to another orientation of spatial axes of the new frame. The time component appears at the Lorentz transformation of projections $\left(A_{1}, A_{2}\right)$ to a direction of motion of the new frame of reference. Propagators of photons will include covariant expressions:

$$
e_{\mu}^{-} e_{v}^{+} A\left(\Lambda x^{\prime}\right) A^{*}(\Lambda x)=e_{\mu}^{a} e_{v}^{b} A_{a}\left(\Lambda x^{\prime}\right) A_{b}(\Lambda x)=A_{\mu}\left(x^{\prime}\right) A_{v}(x) .
$$

Since the tetrad vectors $\left(e_{\mu}^{1}, e_{\mu}^{2}\right)$ are constant and purely kinematical factors, they lead only to kinematical corrections to the quantization procedure and do not change main physical conclusions.

\subsection{Complex scalar and vector fields}

At quantization of rotations of a complex scalar and vector fields, unlike of photons at which field vectors rotate in the real space, it is necessary to study rotations in a complex space of field variables - in an isotopic space. The Lagrangian of the scalar field $\phi=\phi_{1}+i \phi_{2}$ in the minimal form:

$$
L=\int d^{3} x\left(\partial_{\mu} \phi^{*} \cdot \partial^{\mu} \phi-m^{2} \phi^{*} \phi\right),
$$

leads to the Hamiltonian and the the charge operator:

$$
\begin{aligned}
& H=i \int d^{3} x\left(\pi \pi^{*}+\nabla \phi^{*} \nabla \phi+m^{2} \phi^{*} \phi\right), \\
& Q=i \int d^{3} x\left(\phi^{*} \pi^{*}-\pi \phi\right),
\end{aligned}
$$

where $\pi(x)=\partial_{t} \phi^{*}, \pi^{*}(x)=\partial_{t} \phi$. The general solution of field equations includes the frequency modes of 4-momentum, $k=\left( \pm \omega_{\mathbf{k}}, \mathbf{k}\right) \omega_{\mathbf{k}}=\left(\mathbf{k}^{2}+m^{2}\right)^{1 / 2}$, but then negativefrequency quanta should be replaced to positive-frequency antiparticles.

Since all formalism is similar to the case of a photon field (with addition of mass) there appears a problem on, how we can consider $\omega_{\mathbf{k}}$ as a rotation frequency. In the system described by a complex field $\phi=\phi_{1}+i \phi_{2}$ with the Lagrangian (113) there is a global $U(1)$ symmetry under rotation on angle $\lambda$ in two-dimensional space of fields $\left(\phi_{1}, \phi_{2}\right)::$

$$
\phi^{\prime}(x)=e^{i \lambda Q} \phi(x), \quad \phi^{*}(x)=e^{-i \lambda Q} \phi^{*}(x) .
$$

By analogy with the photon field, for the scalar field, represented as an isovector in two-dimensional field space $\vec{\phi}=\left(\phi_{1} \vec{n}_{1}, \phi_{2} \vec{n}_{2}\right)$, we deal with only by the modes in which isovector rotates by frequency $\omega_{k}$, periodically coinciding by $\phi_{1}$ or $\phi_{2}$. Such rotation of field's isovector, generated by the source, then in real space propagates as field's quantum having a wave vector $\mathbf{k}$.

In the complex representation the decomposition of such field on the normal modes has the same form, but with replacement $A \rightarrow \phi$ and practically with the same formalism. Quantization of normal modes of these rotational waves also will lead to the opposite charged quanta of the complex scalar field. The charge in this case, as an analogue of the helicity, is a projection of the isospin to a normal to the isotopic plane and is defined (up to a constant) by the third component of the isospin: $Q \sim \tau_{3}$. Thus, in the rotatory quantization isospin of the complex scalar field is interpreted as the angular momentum of rotations in isotopic plane with frequency of the field quantum. Quantization of the charge, 
which is "isotopic helicity", and energy appear then as the consequences of isovector's angular momentum quantization.

To two equivalent directions of rotation in field space here correspond two signs of the charge and the $C$-symmetry takes place:

$$
H^{c} \equiv C H C^{-1}=H, \quad Q^{c} \equiv C Q C^{-1}=-Q .
$$

It leads to the charge-conjugate ladder operators:

$$
b_{k}=C a_{k} C^{-1}, \quad b_{k}^{*}=C a_{k}^{*} C^{-1},
$$

which define $C$-conjugate vacuum: $b_{k}\left|0_{b}\right\rangle=0$ and the excited states $\left|n_{b}\right\rangle$. The observables $Q^{c}$ and $H^{c}$ also are expressed through these operators.

Further starting from the frequency decomposition of the field on the rotational modes:

$$
\phi(x)=\sum_{k}\left(a_{k} e^{-i k x}+\beta_{k}^{*} e^{i k x}\right), \quad \phi^{*}(x)=\sum_{k}\left(a_{k}^{*} e^{i k x}+\beta_{k} e^{-i k x}\right),
$$

and applying the same method, as for the photon field, we obtain:

$$
\begin{aligned}
& H=\int d^{3} k\left(a_{k}^{*} a_{k}+\beta_{k} \beta_{k}^{*}\right) \omega_{k}=\int d^{3} k\left(a_{k}^{*} a_{k}+b_{k}^{*} b_{k}\right) \omega_{k}, \\
& Q=\int d^{3} k\left(a_{k}^{*} a_{k}-\beta_{k} \beta_{k}^{*}\right)=\int d^{3} k\left(a_{k}^{*} a_{k}-b_{k}^{*} b_{k}\right) .
\end{aligned}
$$

Thus, the operators observables of the complex scalar field with exact $C$-symmetry are normal-ordered and the energy and charge of the vacuum vanish.

A complex vectors field's momentum decomposition to rotational modes is the same as for a scalar field, but by including the polarization vector $\varepsilon_{\mu k}^{\lambda}$ :

$$
\begin{aligned}
& B_{\mu}(x)=\sum_{\mathbf{k} \lambda}\left(a_{k \lambda} \varepsilon_{\mu k}^{\lambda} e^{-i k x}+\beta_{k \lambda}^{*} \varepsilon_{\mu k}^{\lambda^{*}} e^{i k x}\right), \\
& B_{\mu}^{*}(x)=\sum_{\mathbf{k} \lambda}\left(a_{k \lambda}^{*} \varepsilon_{\mu k}^{\lambda^{*}} e^{i k x}+\beta_{k, \lambda} \varepsilon_{\mu k}^{\lambda} e^{-i k x}\right) .
\end{aligned}
$$

After selecting of independent degrees of freedom, the field equations for three polarizations remain linear and are quantized in any frame of reference independently as three complex scalar fields. Therefore, dropping details, we can present only results for the Hamiltonian and the charge operator of the complex vector field:

$$
\begin{aligned}
& H=\sum_{\lambda} \int d^{3} k\left(a_{k \lambda}^{*} a_{k \lambda}+\beta_{k \lambda} \beta_{k \lambda}^{*}\right) \omega_{k}=\sum_{\lambda} \int d^{3} k\left(a_{k \lambda}^{*} a_{k \lambda}+b_{k \lambda}^{*} b_{k \lambda}\right) \omega_{k}, \\
& Q=\sum_{\lambda} \int d^{3} k\left(a_{k \lambda}^{*} a_{k \lambda}-\beta_{k \lambda} \beta_{k \lambda}^{*}\right)=\sum_{\lambda} \int d^{3} k\left(a_{k \lambda}^{*} a_{k \lambda}-b_{k \lambda}^{*} b_{k \lambda}\right),
\end{aligned}
$$

which also do not contain the zero-point energy and zero-point charge.

Although this result is obtained in a fixed frame, nevertheless, the fact of lack of the zero-point energy and zero-point charge is independent on a frame since the vacuum energy-momentum tensor $\Lambda g_{i k}$ is proportional to a scalar constant $\Lambda$ and if this constant vanishes in one a frame, it disappears in all others too.

The symmetries of complex fields allow only gauge-invariant interactions of the form $\left(\phi^{*} \phi+\phi_{c}^{*} \phi_{c}\right)^{n}$, conserving the charge. At rotatory quantization, on the one hand, energies of quanta are determined by charge operators, and on the other hand, interactions of quanta lead to redistribution of these charges. Since the charge operator is normalordered in all orders of the perturbation theory, any degree of this operator also will be normal-ordered and then the vacuum matrix elements from them disappear. This fact sufficiently reduces a number of diagrams in all orders of the perturbation theory. 


\subsection{Spinor field}

The rotational treatment of frequency of a photon leads also to the rotational treatment of frequencies of fermions and their spin that is very attractive. The Lagrangian, Hamiltonian and charge operator of the spinor field have the form:

$$
\begin{aligned}
L & =\int d^{3} x\left[\bar{\psi} \gamma^{\mu}\left(i \partial_{\mu} \psi\right)-m \bar{\psi} \psi\right], \\
H & =\int d^{3} x \frac{1}{2}\left[\psi^{+}\left(i \partial_{t} \psi\right)-\left(i \partial_{t} \psi^{+}\right) \psi\right], \\
Q & =\int d^{3} x \psi^{+} \psi .
\end{aligned}
$$

The frequency decomposition of the spinor field on rotational modes looks like:

$$
\begin{aligned}
& \psi(x)=m \sqrt{2} \sum_{\alpha p}\left(b_{p \alpha} u_{p}^{\alpha} e^{-i p x}+\beta_{p \alpha}^{+} v_{p}^{\alpha} e^{i p x}\right), \\
& \psi^{+}(x)=m \sqrt{2} \sum_{\alpha p}\left(b_{p \alpha}^{+} u_{p}^{\alpha+} e^{i p x}+\beta_{p \alpha} v_{p}^{\alpha+} e^{-i p x}\right),
\end{aligned}
$$

where the normalization is chosen as: $u_{p}^{\alpha+} u_{p}^{\alpha^{\prime}}=v_{p}^{\alpha+} v_{p}^{\alpha^{\prime}}=\delta^{\alpha \alpha^{\prime}} E_{p} / m$, and $E_{p}=\left(\mathbf{p}^{2}+m^{2}\right)^{1 / 2}$.

The spinor field we quantize by the equal time anti-commutators:

$$
\begin{aligned}
& \left\{\bar{\psi}(\mathbf{x}, t), \psi\left(\mathbf{x}^{\prime}, t\right)\right\}=\delta_{x x^{\prime}}^{3} I_{(4)}, \\
& \left\{\psi(\mathbf{x}, t), \psi\left(\mathbf{x}^{\prime}, t\right)\right\}=\left\{\bar{\psi}(\mathbf{x}, t), \bar{\psi}\left(\mathbf{x}^{\prime}, t\right)\right\}=0,
\end{aligned}
$$

which lead to anti-commutators for separate modes, nonzero from which is:

$$
\left\{b_{p \alpha}, b_{p \alpha}^{+}\right\}=\left\{\beta_{p \alpha}, \beta_{p \alpha}^{+}\right\}=\delta_{p p^{\prime}}^{3} \delta_{\alpha \alpha^{\prime}} .
$$

Then, for the charge and Hamiltonian of the free spinor field we obtain:

$$
\begin{aligned}
& H=\sum_{\alpha} \int d^{3} p\left(Q_{p \alpha}^{(b)}-Q_{p \alpha}^{(\beta)}\right) E_{p}=\sum_{\alpha} \int d^{3} p\left(b_{p \alpha}^{+} b_{p \alpha}-\beta_{p \alpha} \beta_{p \alpha}^{+}\right) E_{p}, \\
& Q=\sum_{\alpha} \int d^{3} p\left(Q_{p \alpha}^{(b)}+Q_{p \alpha}^{(\beta)}\right)=\sum_{\alpha} \int d^{3} p\left(b_{p \alpha}^{+} b_{p \alpha}+\beta_{p \alpha} \beta_{p \alpha}^{+}\right) .
\end{aligned}
$$

By introducing the charge-conjugate ladder operators:

$$
C b_{p \alpha} C^{-1}=d_{p \alpha}, \quad C b_{p \alpha}^{+} C^{-1}=d_{p \alpha}^{+}, \quad C \beta_{p \alpha} C^{-1}=\alpha_{p \alpha}, \quad C \beta_{p \alpha}^{+} C^{-1}=\alpha_{p \alpha}^{+}
$$

for the charge-conjugate observables we obtain the expressions:

$$
\begin{aligned}
& H^{c}=\sum_{\alpha} \int d^{3} p\left(Q_{p \alpha}^{(d)}-Q_{p \alpha}^{(\alpha)}\right) E_{p}=\sum_{\alpha} \int d^{3} p\left(d_{p \alpha}^{+} d_{p \alpha}-\alpha_{p \alpha} \alpha_{p \alpha}^{+}\right) E_{p}, \\
& Q^{c}=\sum_{\alpha} \int d^{3} p\left(Q_{p \alpha}^{(d)}+Q_{p \alpha}^{(\alpha)}\right)=\sum_{\alpha} \int d^{3} p\left(d_{p \alpha}^{+} d_{p \alpha}+\alpha_{p \alpha} \alpha_{p \alpha}^{+}\right) .
\end{aligned}
$$

The $C$-symmetry conditions for each of independent modes:

$$
H_{p \alpha}^{c} \equiv C H_{p \alpha} C^{-1}=H_{p \alpha}, \quad Q_{p \alpha}^{c} \equiv C Q_{p \alpha} C^{-1}=-Q_{p \alpha},
$$

written down in more detail:

$$
\begin{aligned}
& H_{p \alpha}=\left(Q_{p \alpha}^{(b)}-Q_{p \alpha}^{(\beta)}\right) E_{p}=\left(Q_{p \alpha}^{(d)}-Q_{p \alpha}^{(\alpha)}\right) E_{p}=H_{p \alpha}^{c}, \\
& Q_{p \alpha}=Q_{p \alpha}^{(b)}+Q_{p \alpha}^{(\beta)}=-\left(Q_{p \alpha}^{(d)}+Q_{p \alpha}^{(\alpha)}\right)=-Q_{p \alpha}^{c},
\end{aligned}
$$

give the operator identities:

$$
Q_{p \alpha}^{(\beta)}=-Q_{p \alpha}^{(d)}, \quad Q_{p \alpha}^{(\alpha)}=-Q_{p \alpha}^{(b)},
$$


or

$$
\alpha_{p \alpha} \alpha_{p \alpha}^{+}=-d_{p \alpha}^{+} d_{p \alpha}, \quad \beta_{p \alpha} \beta_{p \alpha}^{+}=-b_{p \alpha}^{+} b_{p \alpha} .
$$

Their substitution into (126) and (130) gives the final expressions for $H$ and $Q$ :

$$
\begin{aligned}
& H=H^{c}=\sum_{\alpha} \int d^{3} p\left(b_{p \alpha}^{+} b_{p \alpha}+d_{p \alpha}^{+} d_{p \alpha}\right) E_{p}, \\
& Q=-Q^{c}=\sum_{\alpha} \int d^{3} p\left(b_{p \alpha}^{+} b_{p \alpha}-d_{p \alpha}^{+} d_{p \alpha}\right) .
\end{aligned}
$$

Here vacuum expectations both from the charge and spatial components of current disappear:

$$
\langle 0|Q(x)| 0\rangle=0, \quad\langle 0|\mathbf{j}(x)| 0\rangle=0 .
$$

Thus, at quantization of the spinor field the zero-point energy and zero-point charge of the vacuum do not arise.

\subsection{Non-Abelian gauge fields and gravitons}

At quantization of the photon field a physical nature of frequencies has appeared as related to rotations of field vectors and this fact has led to the theory without the vacuum zero-point energy in accordance with experiments. The gauge fields are mainly similar and the same result can be expected for other gauge fields too.

Quantization of non-Abelian gauge fields $A_{\mu}^{a}$ (spin 1) and graviton field (spin 2) in the weak field limit is reduced to quantization of two transverse physical degrees of freedom of several vector or tensor fields. The massless gauge fields have an axial symmetry and at circular polarization their free Hamiltonians and helicity operators are diagonal. Therefore, the rotatory quantization of the photon field is applicable to this case also. Taking into account the non-linearity and internal symmetries of these fields does not change main conclusions about a rotational nature of their frequencies and lack of the vacuum zero-point energy. Really, non-linear contributions are proportional to the coupling constant $g$ both in the self-interaction terms and the covariant derivatives. Therefore, at the weak field approximation $g^{2} \ll 1$ we can neglect these terms and quantize the gauge fields and the graviton field as sets of independent photon degrees of freedom.

In the case of the graviton field an effective coupling constant is weak up to the Planck energy where the gravitational radius of a particle becomes equal to its wavelength and appears a strong gravitational redshift of frequencies of quantum fluctuations. Rapidly decreasing of frequencies at the Planck length because of freezing of proper times with respect to the "distant observer's time", leads to the negligibility and insufficiency of contribution of nonlinear terms [5].

Since the lack of the zero-point energy of a free field follows from the rotational nature of periodicity and frequencies of sources and their quanta, consequently, the additional interactions cannot change it. All contributions to the vacuum energy density from interactions will be proportional to their coupling constants and disappear in the weak field limit (taking into account the cutting at the Planck length), whereas a true zero-point energy does not depend on these factors.

The conclusion that at rotatory quantization of gauge fields and graviton field in one a frame of reference, in the transverse gauge and in the weak-field approximation the zero-point vacuum energy disappears, holds for all frames of reference and for all gauges because of gauge and Lorentz invariance of the vacuum.

Let's note that quanta of non-Abelian fields, unlike photons, carry out the charge of the field and at their quantization the neglecting of a time component of the field vector 
should be carried out by introduction of corresponding compensating contributions from longitudinal component of the field, which however also is not essential for the fact of lack of zero-point energy of this field.

All these, of course, concern to systems without vacuum condensates (or at least regions without them) and to situations where non-perturbative effects do not dominate. Contributions of condensates and topologically nontrivial solutions in the vacuum energy will be considered in forthcoming publication, but they also obviously do not change the conclusions concerning the free fields.

\section{Quantum fields with natural regularizations}

\subsection{Temporary regularization of integrals on trajectories}

In quantum mechanics wave functions $\psi(x, t)$ and $\psi\left(x^{\prime}, t^{\prime}\right)$ are related as:

$$
\psi\left(x^{\prime}, t^{\prime}\right)=\int K\left(x^{\prime}, t^{\prime} ; x, t\right) \psi(x, t) d x(t),
$$

where the amplitude of transition probability $K\left(x^{\prime}, t^{\prime} ; x, t\right)$ is a sum on amplitudes of transition probabilities of all possible alternative classical trajectories connecting the events $(x, t)$ and $\left(x^{\prime}, t^{\prime}\right)$. It can be presented in the form of a path integral, which at square dependence of the Lagrangian $L$ on difference of coordinates has the form [1]:

$$
K\left(x^{\prime}, t^{\prime} ; x, t\right)=\lim _{\Delta t \rightarrow \varepsilon} \prod_{i=1}^{N-1} \int \frac{d x\left(t_{i}\right)}{\sqrt{2 \pi i \Delta t / m}} \exp \left\{i L\left[x\left(t_{i+1}\right)-x\left(t_{i}\right), x\left(t_{i}\right)\right] \Delta t\right\},
$$

where $N=\left(t^{\prime}-t\right) / \Delta t$. Here the measure of the path integral is proportional to $\Delta t^{-1 / 2}$ and, therefore, the transition to very small intervals $\Delta t \rightarrow \varepsilon$ should be performed only after all spatial integrations, i.e. after summation over all alternatives at the given $\Delta t$. This means that quantum-mechanical trajectories $x(t)$ have no time derivatives in usual sense since mean square shifts along them are proportional to $\Delta t$ :

$$
\left\langle[x(t+\Delta t)-x(t)]^{2}\right\rangle \sim \Delta t .
$$

Thus, at quantization of any system time should be divided into relatively small intervals $\Delta t_{i}$, during which trajectories in the path integral (136) are averaged and represented as smooth classical trajectories. At calculation of values of observables, containing time derivatives, at first it is necessary to carry out all averagings at finite $\Delta t$ and only then to pass to the limit $\Delta t \rightarrow \varepsilon$. The formalism of quantum mechanics is constructed so that observables remain finite.

The necessity to regularize the transition amplitudes by averaging during small time intervals $\Delta t$ with approximation by classical trajectories is one of basic physical properties of quantum mechanics in any of its formulations, and the path integrals only clearly reveal this fundamental fact.

\subsection{Field as continuous limit of a chain of harmonic rotators}

In quantum mechanics for transition from coordinate representation to momentum one the system should be placed in a box of relatively large volume $V=L^{3}$ and observations should be made at rather large time $T$. As a result, the momenta and energies in a frequency decomposition of wave function become discrete, and fluctuations of very small frequencies become excluded. Large volume, thus, enters a natural infrared regularization, however does not restrict very high frequency fluctuations. 
In the classical field theory it is known a similar procedure of consideration of a field in a large volume when wavelengths of standing waves become discrete. In this case normal modes of the field are similar to harmonic oscillators and, therefore, the method is called as decomposition of the field on oscillators.

At more physical approach to this procedure the field along each of spatial axes was represented as a chain of $N$ connected harmonic oscillators with subsequent taking the limit $N \rightarrow \infty$. At finite $N$ and finite length of a chain the chain's step $L$ is also finite $a=L / N$ and a continuous limit means $a \rightarrow 0$. However, at field quantization each of oscillators enters the zero-point energy, so in the continuous limit the vacuum energy will be infinite.

In the previous part of the paper the field was represented as a chain of the connected harmonic rotators normal modes of which have no zero-point energy and therefore do not change the vacuum energy, thus such theory remains consistent even in the formal continuous limit.

\subsection{Averaging of field variables in small cells}

In the field theory, both in classical and quantum, for not operating with point charges and correctly enter field variables, it is necessary to average latters in spatial cells of relatively small size $\Delta V_{j}$. As a result, there are upper frequency and wave vector in the frequency decomposition of fields and the averaging is performed on fluctuations of small wavelengths $\lambda \leq \lambda_{N^{\prime}}=\sqrt[3]{\Delta V_{i}}$ and high frequencies $\omega \geq \omega_{N}=2 \pi / \Delta t_{i} . \Lambda_{\Delta x}=2 \pi / \Delta x$.

Thus, the definition of a full set of states is related with the fixed size of the cell at changing the cell's size, we change the full set's number of states too. Therefore, the summation on energy-momentum and summation on spacetime coordinates become not commutative before elimination of the divergences related by high frequencies.

During small times $\Delta t_{i}$ and in small cells $\Delta V_{j}$ the field's evolution is described classically and in the Lagrangians there appear bare masses, charges and field variables obtained by averaging over high-frequency fluctuations in these spacetime cells.

The acts of interaction $H_{I i}(t)$, occurring in time $\Delta t_{i}$ in cells $\Delta V_{j}$, also are described classically, the field is free before and after of each interaction. Then an interaction constant in $\bar{H}_{I}$ appears as an effective constant «renormalized» by taking into account such fluctuations and interactions.

Here only interactions with longer waves $\lambda>\lambda_{N^{\prime}}$ and smaller frequencies $\omega<\omega_{N}$ remain unaccounted. The interactions averaged in cells and small times:

$$
\bar{H}_{I}(t)=\sum_{j} \Delta V_{j} \cdot \bar{H}_{I}\left[\phi_{j}(t, \mathbf{x}), \phi_{j \pm s}(t, \mathbf{x})\right],
$$

can include $\phi_{j \pm s}$, the values in the neighbor cells, defining gradients.

Thus, if the large volume enters the infrared cutting, the small cells regularize the high energy and momentum of modes.

\subsection{Natural regularizations of quantum fields and renormgroup}

At choosing the bare Lagrangian density of scalar field in the form:

$$
L\left[\varphi_{0}, m_{0}, \lambda_{0}, \Lambda_{0}\right]=\frac{1}{2} \partial_{\mu} \varphi_{0}^{*} \cdot \partial^{\mu} \varphi_{0}-\frac{m_{0}^{2}}{2} \varphi_{0}^{*} \varphi_{0}-\frac{\lambda_{0}}{4 !}\left(\varphi_{0}^{*} \varphi_{0}\right)^{2}-\Lambda_{0},
$$


just is result of averaging in small cells, since the bare field $\varphi_{0}$, bare values of mass $m_{0}$, interaction constants $\lambda_{0}$ of quanta and vacuum energy density of the field $\Lambda_{0}$ are obtained by averaging over all fluctuations in spatial cells $\Delta V_{j}$ during time $\Delta t$. Thus, just definition of the bare Lagrangian (139) contains the approximation. The path integral contains contributions only of those quanta the frequency which is less than upper frequency $\omega_{\max }=\alpha \omega_{0}$ with $\omega_{0}=2 \pi / \Delta t$ and $\alpha<1$.

Let's note that contributions of quanta of higher than $\omega_{\max }$ frequencies are not simply rejected, but they are taken into account in the bare values of the field variables and the constants in the Lagrangian (139), which therefore become dependent on $\Delta t$ and $\Delta V_{j}$ , and thus from $\omega_{\max }$ too. But, since the physical Lagrangian $L[\varphi, m, \lambda, \Lambda]$ does not depend on the cells, field quantization assumes division of two kind contributions to it in the form:

$$
L[\varphi, m, \lambda, \Lambda]=L\left[\varphi_{0}, m_{0}, \lambda_{0}, \Lambda_{0} ; \Delta t, \Delta V_{j}\right]+L\left[\delta \varphi_{0}, \delta m_{0}, \delta \lambda_{0}, \delta \Lambda_{0} ; \Delta t, \Delta V_{j}\right]
$$

where the bare Lagrangian expresses the contributions of depending on cells fluctuations of fields and interaction corrections with higher than $\omega_{\max }$ frequencies i.e. $\omega>\omega_{\max }$, and the Lagrangian of counterterms - the contributions with smaller frequencies $\omega<\omega_{\max }$.

Thus, since we start with the bare Lagrangian, it can be expressed through the physical Lagrangian as:

$$
\begin{aligned}
L\left[\varphi_{0}, m_{0}, \lambda_{0}, \Lambda_{0} ; \Delta t\right] & =\frac{1}{2} \partial_{\mu} \varphi \cdot \partial^{\mu} \varphi-\frac{m^{2}}{2} \varphi^{2}-\frac{\lambda}{4 !} \varphi^{4}- \\
& -\Lambda-L\left[\delta \varphi_{0}, \delta m_{0}, \delta \lambda_{0}, \delta \Lambda_{0} ; \Delta t\right],
\end{aligned}
$$

where the dependence on $\Delta t$ is provided by the Lagrangian of counterterms.

In the former formulations of the standard QFT the action function with the bare Lagrangian has been defined not before, but after the limiting process of transition to smallest sells. As the result, in the counterterms there had appeared the divergent contributions, although in renormalizable theories they were cancelled artificially by introduction of counterterms. Now up to limiting transition to the Planck scale spacetime sells $\Delta t \rightarrow \tau_{p}, \Delta V \rightarrow l_{p l}^{3}$, where all quantum fluctuations become frozen, in the renormalized theories the counterterms and the growing loop contributions to the physical Hamiltonian remain finite and consequences of it we will study below.

Here we will note only differences of the new treatment from the standard one. At a series expansion of the $S$-matrix into powers of the interaction Hamiltonian at first we calculate the matrix elements at a fixed sell with $\Delta t \gg \tau_{p}, \Delta V \gg l_{p l}^{3}$ and only then we pass to the limit $\Delta t \rightarrow \tau_{p}, \Delta V \rightarrow l_{p l}^{3}$. By evaluating time-ordered products in the $S$-matrix and turning into momentum representation, we come to ordinary rules of diagram techniques, but with regularized contributions. Separating from the integrals the parts growing at reduction $\Delta t$, we consider them as quantum corrections to the bare Lagrangian. 
Other parts are quantum corrections which lead to measurable effects. The same procedure we perform by spatial integration in the cells.

As a result, integrals in each order of the perturbation theory are finite and they can be replaced by simpler ones cutted at Planck energy $\omega_{k}<\Lambda_{p l}$. Thus, the methods of regularization entered earlier have a meaning in that sense in what may be approximations of the exact and finite sums of the perturbation theory with the correct transition to small times and distances.

One of main properties of fractal structures is the similarity when a part of any fractal structure looks like as the whole structure and a part of this part also repeats the same. A trajectory of the particle at Brownian motion and quantum-mechanical trajectories in the path integrals have the same property of similarity [1]. Thus, at the last examples a role of the characteristic size played smoothed parts of the trajectory passing by a particle during a time slice $\Delta t$. At decreasing the time slice:

$$
\Delta t \rightarrow \Delta t / n,
$$

there appear the same stochastic trajectories as before and so on up to very small temporal slices. The matrix elements for observables in quantum theory, beginning from some small value, become practically independent on further reducing of the sells.

In QFT the similar symmetry of matrix elements has been discovered earlier, called renormgroup, when in the renormalized theories change of a subtraction point $p^{2}=\mu^{2}$ on external momenta in loops with redefinition of parameters of the Lagrangian does not change observing effects [2,3]. Since at time regularization of quantum trajectories there is a characteristic energy $\mu_{\Delta t} \sim 1 / \Delta t$, in diagrams this size plays a role of physically reasonable point of subtraction. The same procedure should be applied to the regularization in the spatial cells. The fact that cell regularization has the similarity property with natural subtraction point $\mu_{\Delta t}$ allows us to consider the renormgroup as the empirically discovered form of the cell regularization.

As the result, the renormgroup becomes a fundamental symmetry of QFT as following from its first principles and describing the property of similarity of the fractal quantum-mechanical trajectories for the field degrees of freedom and renormalizability of fields at the sell regularization. The renormalizability thus expresses the invariance under new kind of spacetime symmetry of quantum systems - renorm-invariance under the size of spacetime cells.

\subsection{Gravitational freezing of fluctuations at the Planck scale}

Regularization of loop contributions averaging in cells of space and time assumes removal of this regularization by reduction of the sizes of cells. Earlier this stage led to internal contradictions since the cells were reduced up to a point and fluctuations of arbitrary large energy and momentum were predicted. In the developed approach the fields are defined not only by averaging in relatively small cells, but also the size of cells can decrease only up to an admissible size - the Planck length $l_{p l}=\left(G \hbar / c^{3}\right)^{1 / 2}$.

There were many attempts to prove that $l_{p l}$ is lower limit for the size of cells, but a physical mechanism for such cutting was unclear and this has made it arbitrary one as cutting at any other scale. 
In the papers $[4,5]$ it was indicated such physical mechanism expiring growth of frequencies of fluctuations at $l_{p l}$, which is not less fundamental than quantum fluctuations. It is the well known gravitational delay of proper times in terms of the world time (in terms of which the $S$-matrix is defined) and which appears as gravitational redshift of frequencies of quanta.

Thus, at quantization of matter fields the action function must include that external gravitational field, with the metrics $g_{\mu \nu}$, accompanying the large energy and momentum fluctuations in the loop diagrams of QFT. This external gravitational field enters into the action function covariantly in the form of the Einstein-Gilbert action:

$$
S=\int d^{4} x \sqrt{g}\left(-\frac{1}{\kappa} R+L_{m}\right)
$$

where $R$ is the Ricci tensor, $g$ is determinant, $\kappa=8 \pi G / c^{4}, G-$ is the gravitational constant, $L_{m}$ - is the Lagrangian of matter fields with all their interactions, including interaction with the external gravitational field too. At the action function variation (143) under the metrics, the Einstein equations, containing the energy-momentum tensor $T_{\mu \nu}$ in the right hand side, should be added to the field equations. They include contributions not only the bare Lagrangian $L_{m}$, but also the perturbation theory series renormalizing parameters of the initial Lagrangian.

However, here it is necessary to specify what we are understand as the contributions to the energy-momentum tensor generating the external gravitational field. In the loop diagrams of the covariant perturbation theory of QFT, as an energy of a particle at intermediate state it has been taken formally $p_{0}$, the time component of conserved 4momentum $p_{\mu}=\left(p_{0}, \mathbf{p}\right)$, where in general case $p_{0}$ does not equal to the physical energy of quanta $E_{\mathbf{p}}=\left(\mathbf{p}^{2}+m_{0}^{2}\right)^{1 / 2}$. At such definition of energy of particles in the one-loop diagram the total «energy» of particles in the loop is restricted by the initial state's total «energy» $p_{0(i n)}=p_{0(1)}+p_{0(2)}$, which is many orders less than the Planck energy. Earlier this interpretation led to the illusion that at loop integrations there will be no noticeable gravitational effects.

In fact, a contribution of intermediate states to the physical energy of a particle on the surface of energy $E_{\mathbf{p}}$ is given by diagrams of the proper energy which grow with the cutting energy due to the momentum integration and, therefore, their contribution lead to the growing gravitational effects.

Let's notice that the component $p_{0}$, formally expressing the time translation invariance, not only breaks the relativistic relation between energy and momentum, but it also has no two other main properties of energy in GR - is not positive-defined and is not a source of gravitational field. At the same time, $E_{\mathbf{p}}$, the physical energy of quanta determined through 3-momenta by the relativistic relation, is positive-defined and, in addition, appears as a source of gravitational field $\left(T_{00} \Delta V \sim E_{\mathbf{p}}\right)$, thus it has all required properties of energy with the exception of conservation. Thus, the $E_{\mathbf{p}}$ not only fluctuates in loops, as required by quantum mechanics where the uncertainty relation $\Delta E \Delta t \geq \hbar / 2$ leads to the fluctuations of physical energy with $\Delta E \neq 0$ in the interval $\Delta t>0$, but also 
gravitates according to GR where the influence of the physical energy to the metrics is inevitable.

Thus, at any world time moment $t$ the total physical energy of particles in the loop of order $2 E_{\mathbf{p}}$ creates the external gravitational field around the center of inertia which in simplest case is given by the Schwarzschild metrics. Other metrics, containing contributions of charges and spins, appear as additions to the main contribution from energy and, therefore, for estimations of main contributions it is enough to consider simplest metrics of GR. This metrics leads to the redshift of the physical energy of quanta:

$$
\bar{E}_{\mathbf{p}}=g_{00}^{1 / 2}(r) E_{\mathbf{p}, r}
$$

and $\bar{E}_{\mathbf{p}}$ acts as a source of external gravitational field of particles in the loop. For states with two particles in the loop (one-loop diagram) a time component of the metrics in the center of inertia frame takes the form:

$$
g_{00}(r)=1-2 G \cdot 2 \bar{E}_{\mathbf{p}} / r .
$$

The field operators and their modes in the frequency decomposition are defined on global hypersurfaces of simultaneity of distant observers $t=$ const. and are defined through $\bar{E}_{\mathbf{p}}$

$$
\phi(\mathbf{r}, t)=\sum_{\mathbf{p}}\left[a_{\mathbf{p}}\left(\bar{E}_{\mathbf{p}}, \mathbf{r}\right) e^{-i \bar{E}_{\mathbf{p}} t}+b_{-\mathbf{p}}^{*}\left(\bar{E}_{\mathbf{p}}, \mathbf{r}\right) e^{i \bar{E}_{\mathbf{p}} t}\right]
$$

The Planck scales, the energy $\Lambda_{p}$ and the distance $l_{p}$, are defined by the condition that the gravitational radius of a two-particle system $r_{g}=2 G \cdot 2 \bar{E}_{\mathbf{p}} / c^{4}$ is of order of wavelength of quantum fluctuations of particles $h c / \bar{E}_{\mathbf{p}}$ (here we show $c$ and $h$ ). The total energy of such system $2 \bar{E}_{\mathbf{p}}$ is of order of $\Lambda_{p}$ :

$$
\begin{gathered}
\frac{2 G \cdot 2 \bar{E}_{\mathbf{p}}}{c^{4}} \simeq \frac{h c}{\bar{E}_{\mathbf{p}}}, \\
2 \bar{E}_{\mathbf{p}} \simeq \sqrt{h c^{5} / G}=\Lambda_{p}=h v_{p l}, \quad v_{p l}=\sqrt{c^{5} / G h}
\end{gathered}
$$

Since the redshift of $\bar{E}_{\mathbf{p}}$ in the gravitational field of particles in the loop is small enough at distances larger $\sim 3 l_{p l}$, already at $r \geq 3 l_{p l}$ and energies $\bar{E}_{\mathbf{p}} \leq 0.3 M_{p l}$ we can use the standard formalism of QFT in flat spacetime. A contribution to amplitudes of fluctuations in the interval $l_{p l} \leq r \leq 3 l_{p l}$ is also small due to strong redshift of $\bar{E}_{\mathbf{p}}$ and leads only to a small correction to the main contribution of the area $\infty \geq r \geq 3 l_{p l}$.

Really, a radius $r$ of localization region of quanta is related by their wavelength as $r \geq 2 \lambda=2 c / v$. In other side, the gravitational radius is proportional to the total energy of the two-particle system which is of order $\sim 2 v$ in terms of the coordinate frequency and therefore:

$$
v=v_{0} \sqrt{1-\frac{r_{g}}{r}} \approx v_{0} \sqrt{1-\frac{2 G h v}{c^{4}} \frac{v}{c}}=v_{0} \sqrt{1-\frac{2 v^{2}}{v_{p l}^{2}}},
$$

where $v_{p l}=\sqrt{c^{5} / G h}-$ is the Planck frequency. This gives a relationship between $v_{0}$ and $v$ : 


$$
v \approx \frac{v_{0}}{\sqrt{1+2 v_{0}^{2} / v_{p l}^{2}}},
$$

As we see, at arbitrary high proper frequency $v_{0} \gg v_{p l}$ the observing coordinate frequencies will not exceed the Planck frequency $v<v_{p l}$.

Thus, the external gravitational field of the loop diagrams restricts the frequencies for the external observer by the Planck frequency. The quanta freeze as strongly, as high their proper frequency and this leads to the effective cutting of the loop integrals at $l_{p l}$.

\subsection{Rotatory quantization and the Planck scale cutting of fields}

Former regularizations of loop contributions in QFT were auxiliary and artificial operations since they did not based on the phenomena naturally cutting energies in loops, moreover the cutting of the energy-momentum 4-vector was relativistically noninvariant operation. Therefore regularizations were removed after calculation observed by obvious or implicit aspiration of energy of cutting to infinity. As physical have been considered the renormalized models where the observables depended on cutting energy through small number of parameters and their replacement by empirical values gave finite predictions.

Gauge theories were renormalized and led to the successful description of interactions in particle physics. But theoretically calculated values of constants of the theory (masses and charges) diverges and the problem of divergences remained in the hidden form. The theory of gravitation following from GR was non-renormalizable because of the dimensional interaction constant. Therefore if to follow the renormalizability criterion, GR should be changed, thus the main efforts for the last sixty years were spent for searching of the generalizing GR renormalizable models of gravitation.

In fact, in the Planck energy scales, where the theory must include effects of the external gravitational field, former two arguments because of which regularizations in loops were considered artificial, lose force. Moreover, the renormalizability criterion becomes as auxiliary one.

On the one hand, as soon as physical energy of any particle on the surface of energy $E_{\mathrm{p}}$ becomes near the Planck energy, it appears a strong external gravitational field around it which slows down proper times of quanta in terms of world time $t$ of distant observers, and appears as strong redshift of proper frequencies. Since the $S$-matrix is constructed in terms of world time $t$, the proper frequencies of quanta in amplitudes will be with so strong redshift that coordinate frequencies higher the Planck frequency do not contribute to the amplitudes and here it takes place their natural gravitational regularization.

On the other hand, the energy of such gravitational regularization is the Planck energy $\Lambda_{p l}$ containing the invariant world constants and, therefore, the regularization with this constant is not only natural, but it is relativistically-invariant too.

Let's note that the methods of quantum field theory in curved spacetime are necessary only for quanta in the field of macroscopic bodies where their trajectories are distorted by a strong field throughout very large number of wavelength of the quanta. However, in the field of microscopic sources of order $l_{p l}$ the relativistic effects of gravitation are small already at distances from a source in few wavelength and, therefore, throughout almost all trajectory quanta can be described as in flat spacetime. Restrictions from relativistic effects of gravitation concern only the processes of emission and absorption of quanta and those several small cells where they occur. 
Thus, the existence of a localization limit of fields in small cells and regularization of loops are related by the fundamental physical reason - by closeness to the gravitational radius of quanta $r_{g}=2 G\left(2 \bar{E}_{p}\right) / c^{4}$ about $l_{p l}$, which leads to freezing of fluctuations and strong redshift of frequencies. The loop divergences in QFT were artificial and they are absent at following to the principles of QFT and GR, since a flat background there is only in the region $\infty \geq r \geq 3 l_{p l}$, while at $l_{p l} \leq r \leq 3 l_{p l}$ the external gravitational field of particles in the loops, slowing down proper times, effectively cuts off all coordinate frequencies.

After the stated above two modifications, i.e. quantizations of rotational modes of fields and averaging in cells with gravitational freezing of fluctuations at $l_{p l}$, the formalism of standard quantum field theory generally preserves, but becomes mathematically correct and, moreover, sufficiently simplifies. The normal ordering arises naturally due to the rotational nature of frequencies, and the invariant regularization - because of the gravitational redshift of frequencies at $l_{p l}$, so the theory leads only to finite expressions for observables. In the theory already there are no inconsistent or doubtful hypotheses, the large class of vacuum and loop diagrams disappears, and calculations of loop integrals become simpler because of the universal cutting energy. The renormalizability from the category of postulates passes into the category of consequences of the formalism and its role is limited only by the energies lower than the Planck energy. Further there appear following simplifications.

Firstly, although the introduction of cells is supposed initially, but in calculations they are not necessary because of smallness of $l_{p l}$ and it is possible to keep convenience of the standard technics of calculations by entering only the cutting at the Planck scales.

Secondly, the intermediate regularizations can be entered also by cell's size of order of measurement's error and the cutting at $l_{p l}$ appears only as lower limit. In the renormalizable theories the loop corrections grow with energy of cutting slowly and remain small even at the gravitational regularization limit. Such theories are finite, the application of the perturbation theory to them is mathematically correct since the corrections are always really small and the perturbation theory series are convergent.

Thirdly, at cell-like regularization up to $l_{p l}$ non-renormalizable theories also become finite though loop corrections to them can be small or large to be of order of the Planck scale. This does, on the one hand, the first, in particular gravitation, physically reasonable when very large value of a loop integral is compensated by very small interaction constant. On the other hand, fundamental fields with ordinary constants of interaction, such as scalar fields, leading to too large contributions at $l_{p l}$, are excluded and they my be associated by composite objects only.

Fourthly, if for usual applications a choice between two determinations of energy for intermediate particles, $p_{0}$ and $E_{\mathbf{p}}$, is a question of convenience of mathematical calculations, however at consideration of energy as a source of gravitational field the choice is not arbitrary since it changes a physical sense and leads to different physical consequences. If we will consider as energy $p_{0}$ then at intermediate states the gravitational effects will absent since the total «energy» of particles in the loop is equal to the total energy of an initial state. In Einstein's equations the stress tensor, in addition of interaction 
energies, includes the physical energy of free quanta $E_{k}=\omega_{k(1)}$, which is non conserving and in intermediate states of the loop diagrams there is an integration over it up to the regularization energy. Therefore, at describing of quantum fluctuations the influence of the external gravitational field generated by these fluctuations is essential and at adequate taking into account of their backward influence on the particles they provide natural cutting the loop integrals at high energies.

Thus, the loop contributions are finite both in one-loop and multiloop diagrams, and are small in the renormalizable theories with weak interaction constant. In them for obtaining a main part of contributions it is enough to consider that cutting is sharp and happens at the Planck energy $\Lambda_{p l}$. At more precise numerical calculations a difference will small, smaller on two or three orders, with respect to the main contribution. Convergence of the perturbation theory series depends on a concrete model and expresses a possibility to apply the perturbation theory to this model only, so this question also becomes technical.

Models in which interactions are carried out by gauge fields are renormalizable and in them the loop integrals grow with energy logarithmically. As a result, if the perturbation theory is fair for tree diagrams, the contribution of loops to the observables is small up to $\Lambda_{p l}$ and at gravitational regularization the perturbation theory series converges and is finite.

At the standard proof of renormalizability of gauge fields in all orders of the perturbation theory is was shown [1-3] that at subtraction on a surface of energy $p_{(0)}^{2}=m^{2}$ the growing terms in full propagators and vertex functions are equivalent to the multiplicative renormalizations:

$$
\tilde{S}_{F}=Z_{2}^{-1} S_{F}^{\prime}, \quad \tilde{D}_{F}=Z_{3}^{-1} D_{F}^{\prime}, \quad \tilde{\Gamma}_{\mu}^{\prime}=Z_{1} \Gamma_{\mu} .
$$

Here it is shown also that all growing contributions are contained only in the renormalization constants $Z_{1}=Z_{2}, Z_{3}$, and that if the corrections in these constants are small, then it leads to the converging perturbation theory series.

Since at a combination of the cell and gravitational regularizations the constants $Z_{i}$ are finite, the loop corrections are small, then the proofs of the renormalizability of gauge fields in all orders of the perturbation theory become simpler, being physically consistent and mathematically correct ones.

Diagrams with corrections growing as degree of energy belong generally to theories with scalar fields and quantum gravity. In the last one they are very small up to $\Lambda_{p l}$, and then are cut off by the gravitational redshift of frequencies, so as a result their contributions are small also and quantum gravity becomes effectively renormalizable.

\section{Particle physics: rotatory quantization and gravitational regularization}

\subsection{Finiteness and consistency of quantum electrodynamics}

Let's turn to consequences of rotational quantization and cell regularization for particle physics both at achieved by observers distances, and up to the gravitational freezing at $l_{p l}$. Let's begin with determination of values of one-loop corrections to quantum electrodynamics (QED) [5]. 
In the spinor electrodynamics the one-loop corrections are logarithmical and at cutting at $\Lambda_{p l}=1.2209 \times 10^{19} \mathrm{GEV}$ include a main factor:

$$
A_{i}=\frac{2 \alpha}{\pi} \ln \left(\Lambda_{p l} / m_{i} c^{2}\right)
$$

where $\alpha^{-1} \simeq 137.036$ and $m_{i}$ is the mass of a charged particle in the intermediate state. For electron with $m_{e} c^{2} \simeq 0.511 \mathrm{MeV}$ it is equal to $A_{e}=0.2394$.

Main contribution to the polarization operator $\Pi\left(q^{2}\right)$ in the limit $q^{2} \rightarrow 0$ gives the electron-positron loop, the correction of which is about $8 \%$ :

$$
\Pi(0) \simeq \frac{1}{3} A_{e} \simeq 0.0798=7.98 \% .
$$

The renormalization constant for the photon propagator then is equal to

$$
Z_{3} \simeq 1-\Pi(0)=0.9202,
$$

which gives for a bare value $\alpha_{0}$ and the correction to the square of charge about 7,4\%,

$$
\begin{gathered}
\alpha_{0}^{-1}=Z_{3} \alpha^{-1} \simeq\left(1-A_{e} / 3\right) \alpha^{-1}=0.9202 \cdot \alpha^{-1} \simeq 126.1015 . \\
\delta e^{2}=e_{0}^{2}-e^{2}=\left(A_{e} / 3\right) e_{0}^{2}=0.0739 e_{0}^{2}=0.0798 e^{2} .
\end{gathered}
$$

The renormalization constants $Z_{2}$ and $Z_{1}$ for the fermion propagator are equal to:

$$
Z_{1}^{(i)}=Z_{2}^{(i)} \simeq 1-\frac{1}{4} A_{i}
$$

For electron a bare mass $m_{e(0)}$ appears as about $85 \%$ of the physical mass $m_{e}$, and a difference between them is about $15 \%$ :

$$
\begin{gathered}
m_{e(0)}=m_{e}\left(1+3 A_{e} / 4\right)^{-1} \simeq 0.8205 m_{e}=0.4193 \mathrm{MэB} \\
\delta m_{e} \simeq m_{e}-m_{e(0)}=0.1522 m_{e}=0.0917 \mathrm{MэB},
\end{gathered}
$$

Other loop diagrams of QED, including multiloop ones too, also remain small and in all cases the gravitational regularization leads to small (in comparison with tree ones) loop contributions to the observables.

Similar values of one loop corrections and bare values of constants for charged particles of the Standard Model are presented in Table 1.

\subsection{Finite Standard Model}

The gauge group $S U(3) \times S U(2) \times U(1)$ of $S M$ at rotational quantization there is no contribution to energy of vacuum from the zero-point fluctuations and the vacuum loop diagrams. It, first, sufficiently simplifies a situation with the ground state of fields, secondly, is in accordance with the experiments that was already discussed in Section 2.1, and, thirdly, excludes a contribution of the vacuum of fundamental fields to the cosmological constant and partly solves this problem.

The energies of states, masses and charges of quanta are related now by their rotational momenta - spins and isospins. What kind is this relation - this is already other question and is a subject of discussions in connection with prospects of unifications. However it is remarkable the fact that rotational quantization reveals a uniform physical

basis for masses, spins and charges of fundamental particles of SM that opens new perspectives for cardinal decreasing of a number of independent parameters of the theory. 
Table 1. Bare values of constants for charged particles of SM and one loop corrections.

\begin{tabular}{|c|c|c|c|c|c|c|c|c|c|c|}
\hline & $\begin{array}{c}m, \\
\mathrm{MeV}\end{array}$ & $A$ & $A / 4$ & $Z_{1}=Z_{2}$ & $\mathrm{P} / 3$ & $Z_{3}$ & $3 \mathrm{~A} / 4$ & $\begin{array}{c}\Delta m, \\
\mathrm{MeV}\end{array}$ & $\Delta m / m$ & $\begin{array}{c}m_{0}, \\
\mathrm{MeV}\end{array}$ \\
\hline & & & & & & & & & & \\
\hline $\mathrm{e}$ & 0,5110 & 0,2394 & 0,0598 & 0,9402 & 0,0798 & 0,9202 & 0,1795 & 0,0917 & 0,8205 & 0,4193 \\
\hline$\mu$ & 105,6584 & 0,2146 & 0,0537 & 0,9463 & 0,0798 & 0,9202 & 0,1610 & 17,0066 & 0,8390 & 88,6518 \\
\hline$\tau$ & 1776,82 & 0,2015 & 0,0504 & 0,9496 & 0,0798 & 0,9202 & 0,1511 & 268,52 & 0,85 & 1508,30 \\
\hline $\mathrm{u}$ & 2,3 & 0,2324 & 0,0581 & 0,9419 & 0,0798 & 0,9202 & 0,1743 & 0,4 & 0,83 & 1,9 \\
\hline $\mathrm{d}$ & 4,8 & 0,2290 & 0,0572 & 0,9428 & 0,0798 & 0,9202 & 0,1717 & 0,8 & 0,83 & 4,0 \\
\hline $\mathrm{s}$ & 95 & 0,2151 & 0,0538 & 0,9462 & 0,0798 & 0,9202 & 0,1613 & 15 & 0,84 & 80 \\
\hline $\mathrm{c}$ & 1275 & 0,2030 & 0,0508 & 0,9492 & 0,0798 & 0,9202 & 0,1523 & 194 & 0,85 & 1081 \\
\hline $\mathrm{b}$ & 4180 & 0,1975 & 0,0494 & 0,9506 & 0,0798 & 0,9202 & 0,1481 & 619 & 0,85 & 3561 \\
\hline $\mathrm{t}$ & 173070 & 0,1802 & 0,0451 & 0,9549 & 0,0798 & 0,9202 & 0,1352 & 23394 & 0,86 & 149676 \\
\hline
\end{tabular}

Another radical simplification of the situation will happen in values of loop corrections to diagrams with external lines. The corrections are finite due to cutting at $\Lambda_{p l}$ and in gauge theories are small due to the logarithmical growth.

In the electroweak theory and quantum chromodynamics the loop corrections contain more rich set of diagrams, but in them also the contributions are reduced practically to differences of decomposition constants multiplied to logarithmical factors of type (152) - At ordinary distances the interaction constants are larger $\alpha$, but at small distances they decrease and the perturbation theory remains applicable up to $\Lambda_{p l}$.

The only field with which in SM there appear problems is a scalar field entered for spontaneous breaking of local gauge symmetries. First, at rotational quantization, for the excluding of the zero-point vacuum energy, the scalar field should has even number of degrees of freedom. This means that the fundamental real valued fields should not exist. Secondly, in it the loop integrals give not only logarithmical, but also growing as a degree of energy contributions which at cutting at $\Lambda_{p l}$ give finite, but very large (about $\Lambda_{p l}^{n}$ ) contributions to the observables. From these two facts follows that or the scalar particles are composite and their field is effective, or there is another, compatible with the known facts, mechanism for the mass generation of SM particles.

Taking into account all these the SM at taking into account the rotational nature of frequencies of quanta of fields and the gravitational regularization at $\Lambda_{p l}$ becomes consecutive due to finiteness of predicted value of observables and compatibility with observations without doubtful procedures.

\subsection{Finiteness and consistency of quantum gravity}

The regularization of loop contributions by a proper external gravitational field with cutting at $\Lambda_{p l}$ will remain for quantum gravity too since the gravitational delay of proper times only amplifies by quantum effects of gravitation.

The dimensional interaction constant in quantum gravity leads to power contributions from graviton loop diagrams. However, unlike a scalar field, here the effective dimensionless interaction constant $G l_{p l}^{2}$ is very small in the most part of the 
energy interval up to $\Lambda_{p l}$, so the loop contributions of quantum gravity, both logarithmical and power ones, remain small even near $\Lambda_{p l}$. As the result, quantum gravity is not only finite, but here it is applicable the perturbation theory and the physical observables may be presented in the form of bare values and small quantum corrections. At decomposition over the effective interaction constant the perturbation theory series are convergent.

\subsection{New perspectives of unification}

Thus, now particle physics no need in additional hypotheses, such as supersymmetry, extended fundamental objects (strings, branes) and higher dimensions with very small radius. Now the strong theoretical reasons for such hypotheses disappear and they are of interest only at the appearance of observational indications which while are absent.

Attempts of unification of three gauge fields of SM $S U(3) \times S U(2) \times U(1)$ into a one were based on a hypothesis that at energies of order $10^{15} \mathrm{GEV}$ the charges of these three gauge fields become of the same order. In fact, in the asymptotics of charges of SM at the gravitational regularization at the Planck energy the curves for charges of electromagnetic, weak and strong interactions cross only in pairs and in three points, the energy scales of which differ to several orders. This shows that within SM the Grand Unification of three fundamental interactions is practically excluded, or there it is required to take into account some additional factors which at this stage are unclear.

Another popular working hypothesis is the hypothesis about the composite nature of SM particles which also enters some scale of energy. Now this direction becomes the most intriguing one, but here it is required to find mechanisms allowing to exclude a large contribution to the mass of a composite particle from its subparticles localized in very small volume.

\section{Conclusion}

The observables of the $C$-symmetric systems, the modes of which are harmonic oscillators in complex representation, should be expressed through two sets of mutually $C$ conjugate ladder operators, but their introduction is non-trivial and the $C$-symmetry conditions lead to some operator identities. These identities lead to automatically normal ordering of the operators in the expressions for observables and the system's ground state does not contain the zero-point energy and zero-point charge.

This fact is in agreement with the generalization of uncertainty relations on nonHermitean operators of canonical variables which include the mean value of the charge operator.

At representation of states of antiquanta as states of quanta with negative energy and frequency (evolving backward in time) a sign of mass also should be negative and then the norm of negative frequency states with becomes positive defined.

In the model of the harmonic rotator the operators of observables are normally ordered because of $C$-symmetry and there is no zero-point energy in the ground state.

Applications of the new formalism to quantization of waves at collective rotations of a one-dimensional chain of harmonic rotators allows to model the fields with gauge and $C$ symmetries, and with spinor excitations.

At rotational quantization of fields, where the frequency of quanta means the rotation frequency of field vectors, their modes are similar to the harmonic rotator. For this reason the operators in the observables are automatically normally ordered, and zero-point 
energy and charge of vacuum do not arise, including the interacting fields. In the covariant perturbation theory the transition to the harmonic rotator quanta is reduced in general case to the existence of $C$-symmetry and normal ordering of operator products. For this reason the agreement between the theory and experiments remains unchanged, moreover, the new approach is free from the zero-point vacuum energy problem. The rotatory quantization of relativistic fields allows to understand the physical nature of spins and charges of particles on a uniform basis by relating them with the angular momenta at rotations of field vectors with a frequency defining the energy of quanta. Moreover, the quantized values of energy, spin and charge of quanta appear as the result of a quantizad value of the angular momenta of the field vectors.

In QFT there is a natural cutting of integrals on energy in the form of cell regularization with the minimal and invariant value of this cutting at $l_{p l}$. The regularization procedures, used in former methods of renormalization, appear as the consequences of the cell regularization. At invariant gravitational regularization because of the gravitational redshift at the Planck energy $\Lambda_{p l}$ the loop corrections of SM remain rather small (except a scalar field), so the perturbation theory becomes mathematically correct and physically consistent.

Modes of a relativistic string do not contain the zero-point energy because of chiral symmetry, then there is no conform anomaly and the spacetime dimension may be arbitrary.

Acknowledgments. In discussion of ideas and results of the paper participated the large number of colleagues to whom I am grateful for the criticism, which allowed to improve the argumentation, and for support. It is especially grateful to L.D. Faddeev for support of the point of view that the lack of the zero-point vacuum energy and possibility of regularization of fields by their external gravitational field will allow to make QFT more consecutive, at least, in case of quantum electrodynamics (added at 30.03.2015).

\section{Appendix. Rotatory quantization of strings without anomalies}

In the last decades radical models based on introduction of non-local fundamental objects - strings and branes - have been widely developed. In the string models a key role plays the zero-point energy of oscillatory modes of strings which was regularized so that there were anomalies and the central charge in the Virasoro algebra. The exclusion of this anomaly fixed the spacetime dimension and restricted states and gauge symmetries. All these properties of the model caused large enthusiasm.

However it was shown in the paper [6] that at more consecutive approach to the string models it is necessary to quantize rotational modes of strings, the chiral symmetry of which then excludes the zero-point energy, so the conformal anomaly too, and thus the string models are well-defined in spacetime of any dimension. This method of quantization is presented below on the example of a closed boson string.

Let's consider the standard action for a boson string:

$$
S=-\frac{T}{2} \int d \tau d \sigma h^{\alpha \beta}(\tau, \sigma) \partial_{\alpha} X^{\mu} \partial_{\beta} X_{\mu} .
$$

Three local symmetries, two reparametrizations and the Weyl dilatation allow to choose three independent components of the 2-metric of the string as: 


$$
h_{\alpha \beta}(\tau, \sigma)=\eta_{\alpha \beta}=\left(\begin{array}{cc}
1 & 0 \\
0 & -1
\end{array}\right),
$$

then the action (160) can be written in the form:

$$
S=-(T / 2) \int d^{2} \sigma \partial_{\alpha} X^{\mu} \partial^{\alpha} X_{\mu} .
$$

The equations of motion for the closed string following from the action (160):

$$
\partial_{\alpha} \partial^{\alpha} X^{\mu}=\left(\partial_{\tau}^{2}-\partial_{\sigma}^{2}\right) X^{\mu}=0
$$

have a general solution in the form of superposition of two independent modes:

$$
X^{\mu}(\tau, \sigma)=X_{R}^{\mu}(\tau-\sigma)+X_{L}^{\mu}(\tau+\sigma) .
$$

We can join the coordinates of these modes into one a complex coordinate:

$$
\begin{aligned}
& Y^{\mu}(\tau, \sigma)=\left[X_{R}^{\mu}(\tau-\sigma)+i X_{L}^{\mu}(\tau+\sigma)\right] / \sqrt{2}, \\
& Y^{\mu^{*}}(\tau, \sigma)=\left[X_{R}^{\mu}(\tau-\sigma)-i X_{L}^{\mu}(\tau+\sigma)\right] / \sqrt{2} .
\end{aligned}
$$

In terms of this coordinate the action and the energy-momentum tensor take a form:

$$
\begin{gathered}
S=-(T / 2) \int d^{2} \sigma\left[\partial_{\alpha} X_{R}^{\mu} \partial^{\alpha} X_{\mu R}+\partial_{\alpha} X_{L}^{\mu} \partial^{\alpha} X_{\mu L}\right]=-T \int d^{2} \sigma \partial_{\alpha} Y^{*} \cdot \partial^{\alpha} Y, \\
T_{\alpha \beta}=-\frac{2}{T \sqrt{-\eta}} \frac{\delta S}{\delta \eta^{\alpha \beta}}=\frac{1}{2}\left[\partial_{\alpha} Y_{\mu}^{*} \partial_{\beta} Y^{\mu}-\frac{1}{2} \eta_{\alpha \beta} \partial_{\alpha^{\prime}} Y_{\mu}^{*} \partial^{\alpha^{\prime}} Y^{\mu}+\text { h.c. }\right]=0 .
\end{gathered}
$$

The traceless of the energy-momentum tensor leads to the constraint equations:

$$
T_{00}=\frac{1}{2}\left(\partial_{\tau} Y^{*} \partial_{\tau} Y+\partial_{\sigma} Y^{*} \partial_{\sigma} Y\right)=0, T_{10}=\frac{1}{2}\left(\partial_{\tau} Y^{*} \cdot \partial_{\sigma} Y+\partial_{\sigma} Y^{*} \cdot \partial_{\tau} Y\right)=0
$$

which give the following relations:

$$
\partial_{\tau} Y^{*} \partial_{\tau} Y=\partial_{\sigma} Y^{*} \partial_{\sigma} Y=0, \quad \partial_{\tau} Y^{*} \cdot \partial_{\sigma} Y=\partial_{\sigma} Y^{*} \cdot \partial_{\tau} Y=0 .
$$

From the equations of motion (163) and the boundary conditions for closed strings:

$$
Y^{\mu}(\tau, \sigma)=Y^{\mu}(\tau, \sigma+2 \pi), \quad Y^{\mu^{*}}(\tau, \sigma)=Y^{\mu^{*}}(\tau, \sigma+2 \pi)
$$

it follows the decomposition of the complex coordinate on frequency modes:

$$
\begin{aligned}
& Y^{\mu}(\tau, \sigma)=\frac{y^{\mu}}{2}+\frac{l^{2}}{2}\left(\pi^{\mu} \tau-\pi^{\mu^{*}} \sigma\right)+\frac{i l}{2} \sum_{n \neq 0} \frac{1}{|n|}\left(\alpha_{n}^{\mu} e^{-2 i(|n| \tau-n \sigma)}+b_{n}^{\mu^{*}} e^{2 i(|n| \tau-n \sigma)}\right), \\
& Y^{\mu^{*}}(\tau, \sigma)=\frac{y^{\mu^{*}}}{2}+\frac{l^{2}}{2}\left(\pi^{\mu^{*}} \tau-\pi^{\mu} \sigma\right)-\frac{i l}{2} \sum_{n \neq 0} \frac{1}{|n|}\left(\alpha_{n}^{\mu^{*}} e^{2 i(|n| \tau-n \sigma)}+b_{n}^{\mu} e^{-2 i(|n| \tau-n \sigma)}\right),
\end{aligned}
$$

where $l^{2}=1 / \pi T$ and $y^{\mu}=x^{\mu} \cdot(1+i) / \sqrt{2}, \pi^{\mu}=p^{\mu} \cdot(1-i) / \sqrt{2}$. From this it also follows:

$$
\begin{aligned}
& \partial_{\tau} Y^{\mu}(\tau, \sigma)=\frac{1}{2} l^{2} \pi^{\mu}+l \sum_{n \neq 0}\left(\alpha_{n}^{\mu} e^{-2 i(|n| \tau-n \sigma)}-b_{n}^{\mu^{*}} e^{2 i(|n| \tau-n \sigma)}\right), \\
& \partial_{\tau} Y^{\mu^{*}}(\tau, \sigma)=\frac{1}{2} l^{2} \pi^{\mu^{*}}+l \sum_{n \neq 0}\left(\alpha_{n}^{\mu^{*}} e^{2 i(|n| \tau-n \sigma)}-b_{n}^{\mu} e^{-2 i(|n| \tau-n \sigma)}\right),
\end{aligned}
$$

Here the proper times $\tau$ on a two-dimensional surface are multiplied only to the positive «frequencies» $|n|$. The sums of two independent contributions of opposite sign of frequency are written separately, where creation operators of positive-frequency quanta are denoted as $\alpha_{n}^{\mu^{*}}$, while the auxiliary operators for negative-frequency quanta as $b_{n}^{\mu^{*}}$. The equal time commutators look like: 


$$
\begin{aligned}
& i\left[\partial_{\tau} Y^{v}(\tau, \sigma), Y^{\mu^{*}}\left(\tau, \sigma^{\prime}\right)\right]=-T^{-1} \eta^{\mu v} \delta\left(\sigma-\sigma^{\prime}\right), \\
& i\left[\partial_{\tau} Y^{\nu^{*}}(\tau, \sigma), Y^{\mu}\left(\tau, \sigma^{\prime}\right)\right]=-T^{-1} \eta^{\mu v} \delta\left(\sigma-\sigma^{\prime}\right) .
\end{aligned}
$$

The substitution of (171) and (172) give us following nonzero commutators for the operators of modes:

$$
\left[\alpha_{m}^{\mu}, \alpha_{n}^{v^{*}}\right]=\left[b_{m}^{\mu}, b_{n}^{v^{*}}\right]=-|m| \eta^{\mu v} \delta_{m n}, \quad i\left[\pi^{\mu}, y^{v}\right]=i\left[\pi^{\mu^{*}}, y^{v^{*}}\right]=-\eta^{\mu v},
$$

where $\alpha_{0}^{\mu}=b_{0}^{\mu}=l \pi^{\mu} / 2$. The conserved currents for translations of coordinates $Y^{\mu}, Y^{\mu^{*}}$ are:

$$
\begin{gathered}
P_{\alpha}^{\mu}=T \partial_{\alpha} Y^{\mu^{*}}, \quad \partial^{\alpha} P_{\alpha}^{\mu}=0, \\
P_{\tau}^{\mu}=(2 \pi)^{-1} \pi^{\mu}+l \sum_{n \neq 0}\left(\alpha_{n}^{\mu} e^{-2 i(|n| \tau-n \sigma)}-b_{n}^{\mu^{*}} e^{2 i(n \mid \tau-n \sigma)}\right), \\
P_{\sigma}^{\mu}=-(2 \pi)^{-1} \pi^{\mu^{*}}-l \sum_{n \neq 0} \frac{n}{|n|}\left(\alpha_{n}^{\mu} e^{-2 i(|n| \tau-n \sigma)}-b_{n}^{\mu^{*}} e^{2 i(n \mid \tau-n \sigma)}\right),
\end{gathered}
$$

The total momentum of the closed string is $(\tau=0)$ :

$$
P^{\mu}=T \int_{0}^{2 \pi} d \sigma \partial_{\tau} Y^{\mu^{*}}=\pi^{\mu} .
$$

The Hamiltonian then takes the form:

$$
\begin{gathered}
H=\int_{0}^{2 \pi} d \sigma\left[\left(P_{\tau}^{\mu} \partial_{\tau} Y_{\mu}+\partial_{\tau} Y_{\mu}^{*} P_{\tau}^{\mu^{*}}\right)-L\right]=T \int_{0}^{2 \pi} d \sigma\left(\partial_{\tau} Y_{\mu}^{*} \partial_{\tau} Y^{\mu}+\partial_{\sigma} Y_{\mu}^{*} \partial_{\sigma} Y^{\mu}\right), \\
H=T \int_{0}^{2 \pi} d \sigma\left(\partial_{\tau} Y^{\mu^{*}} \partial_{\tau} Y_{\mu}+\partial_{\sigma} Y^{\mu^{*}} \partial_{\sigma} Y_{\mu}\right)=2 \sum_{n=-\infty}^{\infty}\left(\alpha_{n}^{\mu^{*}} \alpha_{n \mu}+b_{n}^{\mu} b_{n \mu}^{*}\right) .
\end{gathered}
$$

The left-hand and right-hand modes of the string are independent and a conserved helicity operator of the string, playing the role of a chiral charge, is given by the expressions:

$$
\begin{aligned}
\Lambda & =T \int_{0}^{2 \pi} d \sigma\left[\left(\partial_{\tau} Y_{\mu}^{*}\right) Y^{\mu}-Y^{\mu^{*}} \partial_{\tau} Y_{\mu}\right]=\int_{0}^{2 \pi} d \sigma\left(P_{\tau}^{\mu} Y_{\mu}-Y_{\mu}^{*} P_{\tau}^{\mu^{*}}\right)= \\
& =i\left(\pi^{\mu} y^{\nu}-y^{\nu^{*}} \pi^{\mu^{*}}\right)-i \sum_{n \neq 0} \frac{1}{|n|}\left(\alpha_{n}^{\mu^{*}} \alpha_{n \mu}-b_{n}^{\mu} b_{n \mu}^{*}\right) .
\end{aligned}
$$

There is a symmetry transferring the left-modes to right-modes and backward by chiral charge conjugation ( $C$-symmetry). Let's introduce also the operators $C$-conjugate to the former operators of the string modes:

$$
\beta_{n}^{\mu}=C \alpha_{n}^{\mu} C^{-1}, \quad \beta_{n}^{\mu^{*}}=C \alpha_{n}^{\mu^{*}} C^{-1}, \quad a_{n}^{\mu}=C b_{n}^{\mu} C^{-1}, \quad a_{n}^{\mu^{*}}=C b_{n}^{\mu^{*}} C^{-1} .
$$

Further, by means of these operators the $C$-symmetry requirements can be written in the form:

$$
\begin{aligned}
& H=\sum_{n=-\infty}^{\infty}\left(\alpha_{n}^{\mu^{*}} \alpha_{n \mu}+b_{n}^{\mu} b_{n \mu}^{*}\right)=\sum_{n=-\infty}^{\infty}\left(\beta_{n}^{\mu^{*}} \beta_{n \mu}+a_{n P}^{\mu} a_{n \mu P}^{*}\right)=H^{c}, \\
& \Lambda=-i \sum_{n=-\infty}^{\infty} \frac{1}{|n|}\left(\alpha_{n}^{\mu^{*}} \alpha_{n \mu}-b_{n}^{\mu} b_{n \mu}^{*}\right)=i \sum_{n=-\infty}^{\infty} \frac{1}{|n|}\left(\beta_{n}^{\mu^{*}} \beta_{n \mu}-a_{n P}^{\mu} a_{n \mu P}^{*}\right)=-\Lambda^{c} .
\end{aligned}
$$


Because of independence of modes the $C$-symmetry requirements take place for each mode separately and consequently (at $y^{v}=0$ ):

$$
\begin{aligned}
& H_{n}=\left(\alpha_{n}^{\mu^{*}} \alpha_{n \mu}+b_{n}^{\mu} b_{n \mu}^{*}\right)=\left(\beta_{n}^{\mu^{*}} \beta_{n \mu}+a_{n}^{\mu} a_{n \mu}^{*}\right)=H_{n}^{c}, \\
& \Lambda_{n}=|n|^{-1}\left(\alpha_{n}^{\mu^{*}} \alpha_{n \mu}-b_{n}^{\mu} b_{n \mu}^{*}\right)=-|n|^{-1}\left(\beta_{n}^{\mu^{*}} \beta_{n \mu}-a_{n}^{\mu} a_{n \mu}^{*}\right)=-\Lambda_{n}^{c},
\end{aligned}
$$

or:

$$
\begin{gathered}
\alpha_{n}^{\mu^{*}} \alpha_{n \mu}+b_{n}^{\mu} b_{n \mu}^{*}=\beta_{n}^{\mu^{*}} \beta_{n \mu}+a_{n}^{\mu} a_{n \mu}^{*}, \\
\alpha_{n}^{\mu^{*}} \alpha_{n \mu}-b_{n}^{\mu} b_{n \mu}^{*}=-\beta_{n}^{\mu^{*}} \beta_{n \mu}+a_{n}^{\mu} a_{n \mu}^{*} .
\end{gathered}
$$

By adding and subtracting (185) and (186) for each string mode, we obtain:

$$
\alpha_{n}^{\mu^{*}} \alpha_{n \mu}=a_{n}^{\mu} a_{n \mu}^{*}, \quad \beta_{n}^{\mu^{*}} \beta_{n \mu}=b_{n}^{\mu} b_{n \mu}^{*} \text {. }
$$

Thus, at taking into account (187) the Hamiltonian and the helicity of the string (183) take the form:

$$
H=\sum_{n \neq 0}\left(\alpha_{n}^{\mu^{*}} \alpha_{n \mu}+\beta_{n \mu}^{*} \beta_{n}^{\mu}\right), \quad \Lambda=\sum_{n \neq 0} \frac{1}{|n|}\left(\alpha_{n}^{\mu^{*}} \alpha_{n \mu}-\beta_{n \mu}^{*} \beta_{n}^{\mu}\right),
$$

where the operators of the string modes enter in a normal-ordered form and there is no zero-point energy.

This fact leads to the quantum theory of strings without conformal anomaly, since only the presence of the zero-point energy of string modes was a physical reason for this anomaly. Then the lack of the anomaly does not allow one to fix dimensionality of spacetime. Below this fact will be considered in more detail form.

Let's consider the decomposition on modes of the constraints $T_{\alpha \beta}=0$. For the closed string the constraints are reduced to $\partial_{\tau} Y^{*} \partial_{\tau} Y=\partial_{\sigma} Y^{*} \partial_{\sigma} Y=0$ and $(\tau=0)$ :

$$
\begin{gathered}
L_{m}=\frac{T}{2} \int_{0}^{2 \pi} d \sigma e^{-i m \sigma} T_{00}(0, \sigma)=\frac{T}{4} \int_{0}^{2 \pi} d \sigma e^{-i m \sigma}\left(\partial_{\tau} Y^{*} \partial_{\tau} Y+\partial_{\sigma} Y^{*} \partial_{\sigma} Y\right), \\
L_{m}^{\prime}=\frac{T}{2} \int_{0}^{2 \pi} d \sigma e^{-i m \sigma} T_{10}(0, \sigma)=\frac{T}{2} \int_{0}^{2 \pi} d \sigma e^{-i m \sigma} \partial_{\tau} Y^{*} \partial_{\sigma} Y .
\end{gathered}
$$

In quantum theory the substitution of decomposition on modes gives:

$$
L_{n}=\frac{1}{2} \sum_{p=-\infty}^{\infty}\left(\alpha_{p}^{\mu^{*}} \alpha_{n+p}^{\mu}+b_{p}^{\mu} b_{n+p}^{\mu^{*}}\right) .
$$

For replacement of the second term we will use the $C$-symmetry conditions for each mode:

$$
\begin{aligned}
& L_{n p}=2^{-1}\left(\alpha_{p}^{\mu^{*}} \alpha_{n+p}^{\mu}+b_{p}^{\mu} b_{n+p}^{\mu^{*}}\right)=2^{-1}\left(\beta_{p}^{\mu^{*}} \beta_{n+p}^{\mu}+a_{p}^{\mu} a_{n+p}^{\mu^{*}}\right)=L_{n p}^{c}, \\
& \Lambda_{n p}=(2|p|)^{-1}\left(\alpha_{p}^{\mu^{*}} \alpha_{n+p}^{\mu}-b_{p}^{\mu} b_{n+p}^{\mu^{*}}\right)=-(2|p|)^{-1}\left(\beta_{p}^{\mu^{*}} \beta_{n+p}^{\mu}-a_{p}^{\mu} a_{n+p}^{\mu^{*}}\right)=-\Lambda_{n p}^{c},
\end{aligned}
$$

and obtain:

$$
b_{p}^{\mu} b_{n+p}^{\mu^{*}}=\beta_{p}^{\mu^{*}} \beta_{n+p}^{\mu} .
$$

These relations lead to the normal ordered expression:

$$
L_{n}=\frac{1}{2} \sum_{p=-\infty}^{\infty}\left(\alpha_{p}^{\mu^{*}} \alpha_{n+p}^{\mu}+\beta_{p}^{\mu^{*}} \beta_{n+p}^{\mu}\right) .
$$

For finding an algebra of operator further we will use the relations: 


$$
\begin{gathered}
{\left[\alpha_{m}^{\mu}, \alpha_{n}^{v^{*}}\right]=-|m| \eta^{\mu v} \delta_{m n}, \quad\left[\alpha_{m}^{\mu}, \alpha_{n}^{v}\right]=\left[\alpha_{m}^{\mu^{*}}, \alpha_{n}^{\nu^{*}}\right]=0,} \\
{\left[\alpha_{m}^{\mu}, L_{n}\right]=\sum_{p=-\infty}^{\infty}\left[\alpha_{m}^{\mu}, \alpha_{p}^{v^{*}} \alpha_{n+p}^{v}\right]=-|m| \sum_{p=-\infty}^{\infty} \eta^{\mu v} \delta_{m p} \alpha_{n+p}^{v}=-|m| \alpha_{m+n}^{\mu},} \\
{\left[\alpha_{m}^{i^{*}}, L_{n}\right]=\sum_{p=-\infty}^{\infty}\left[\alpha_{m}^{i^{*}}, \alpha_{p}^{j^{*}} \alpha_{n+p}^{j}\right]=|m| \sum_{p=-\infty}^{\infty} \alpha_{p}^{v^{*}} \eta^{\mu v} \delta_{m, n+p}=|m| \alpha_{m-n}^{\mu^{*}} .}
\end{gathered}
$$

Similar relations take place and for the $C$-conjugate operators. Then we obtain:

$$
\begin{aligned}
& {\left[L_{m}, L_{n}\right]=\sum_{p=-\infty}^{\infty}\left[L_{m}, \alpha_{p}^{\mu^{*}} \alpha_{n+p}^{\mu}\right]=\sum_{p=-\infty}^{\infty}\left[L_{m}, \alpha_{p}^{\mu^{*}}\right] \alpha_{n+p}^{\mu}+\sum_{p=-\infty}^{\infty} \alpha_{p}^{\mu^{*}}\left[L_{m}, \alpha_{n+p}^{\mu}\right]=} \\
& =|n-m| \sum_{p=-\infty}^{\infty}\left(\alpha_{p}^{\mu^{*}} \alpha_{n+m+p}^{\mu}+\beta_{p}^{\mu^{*}} \beta_{n+m+p}^{\mu}\right)=|n-m| L_{n+m} .
\end{aligned}
$$

So, the required algebra of operators is given by the relations:

$$
\left[L_{m}, L_{n}\right]=|n-m| L_{n+m} .
$$

in which, due to the lack of the zero-point energy, there are no anomalous terms.

Thus, the modes of the relativistic boson string do not contain the zero-point energy. At the lack of the zero-point energy of modes and automatically normal ordering, the algebra of operators is normal (there is no central extension in the Virasoro algebra) and the conformal anomaly is absent. For the same reason at quantization of a fermion string, where a chiral symmetry takes place also and there is no zero-point energy, the conformal anomaly does not arise. The lack of conformal anomaly does not allow one to fix the dimensionality $D$ of spacetime, into which the string is embedded, and the string theories can be consistently quantized in a spacetime of arbitrary dimension $D>2$.

\section{References}

1. Bjorken J., Drell S. Relativistic Quantum Theory. 1964.

2. Faddeev L.D., Slavnov A.A. Introd. to Quant. Theory of Gauge Fields. 1988.

3. Weinberg S. Quantum Field Theory. v. 1-3. 1995.

4. Zakir Z. (2011) Teor. Phys., Astrophys. \& Cosmol. 6(1) 1; 6(2) 14; 6(2) 32; 6(2) 50.

5. Zakir Z. (2006) Teor. Phys., Astrophys. \& Cosmol. 1(2) 30.

6. Zakir Z. (2008) Teor. Phys., Astrophys. \& Cosmol. 3(2) 10. 